\title{
Revealing the state space of turbulent pipe flow by symmetry reduction
}

\author{
By A. P. WILLIS ${ }^{1}$ P. CVIT A N O V I Ć $\dot{C}^{2}$ \\ A ND M. A VIL $\mathbf{A}^{3,4}$ \\ ${ }^{1}$ School of Mathematics and Statistics, University of Sheffield, S37RH, U.K. \\ ${ }^{2}$ School of Physics, Georgia Institute of Technology, Atlanta, GA 30332, USA \\ ${ }^{3}$ Max Planck Institute for Dynamics and Self-Organization (MPIDS), \\ 37077 Göttingen, Germany \\ ${ }^{4}$ Institute of Fluid Mechanics, Fridriech-Alexander-Universität Erlangen-Nürnberg, \\ Cauerstrasse 4, 91058 Erlangen, Germany
}

(Printed 9 October 2018)

Symmetry reduction by the method of slices is applied to pipe flow in order to quotient the stream-wise translation and azimuthal rotation symmetries of turbulent flow states. Within the symmetry-reduced state space, all travelling wave solutions reduce to equilibria, and all relative periodic orbits reduce to periodic orbits. Projections of these solutions and their unstable manifolds from their $\infty$-dimensional symmetry-reduced state space onto suitably chosen 2- or 3-dimensional subspaces reveal their interrelations and the role they play in organising turbulence in wall-bounded shear flows. Visualisations of the flow within the slice and its linearisation at equilibria enable us to trace out the unstable manifolds, determine close recurrences, identify connections between different travelling wave solutions, and find, for the first time for pipe flows, relative periodic orbits that are embedded within the chaotic attractor, which capture turbulent dynamics at transitional Reynolds numbers.

\section{Introduction}

The understanding of chaotic dynamics in high-dimensional systems that has emerged in the last decade offers a promising dynamical framework to study turbulence. Here turbulence is viewed as a walk through a forest of exact solutions in the $\infty$-dimensional state-space of the governing equations. In pipe flow, the discovery of unstable travelling waves (Faisst \& Eckhardt 2003; Wedin \& Kerswell 2004), together with glimpses of them in experiments (Hof et al. 2004), has spurred interest in obtaining a description of turbulent flow in terms of the dynamics of a handful of key exact solutions. However, evidence of the relevance of the dynamical system approach to turbulence has so far been mostly provided by studies of plane Couette flow (Gibson et al. 2008, 2009; Halcrow et al. 2009), with the discovery of periodic (Kawahara \& Kida 2005; Cvitanović \& Gibson 2010) and relative periodic orbits (Viswanath 2007) embedded in turbulence playing the key role. In this approach, the dynamics of turbulent flows at moderate Reynolds number $(R e)$ is visualised using equilibrium solutions of the Navier-Stokes equations to define dynamically invariant, intrinsic, and representation-independent coordinate frames (Gibson et al. 2008). The resulting visualisations show the role exact solutions play in shaping turbulence: the observed coherent structures are the physical images of the flow's least unstable invariant solutions, with turbulent dynamics arising from a sequence of transitions between these states. Here the intrinsic low-dimensionality of turbulence stems from the low number of unstable eigendirections for each state. In this picture periodic 

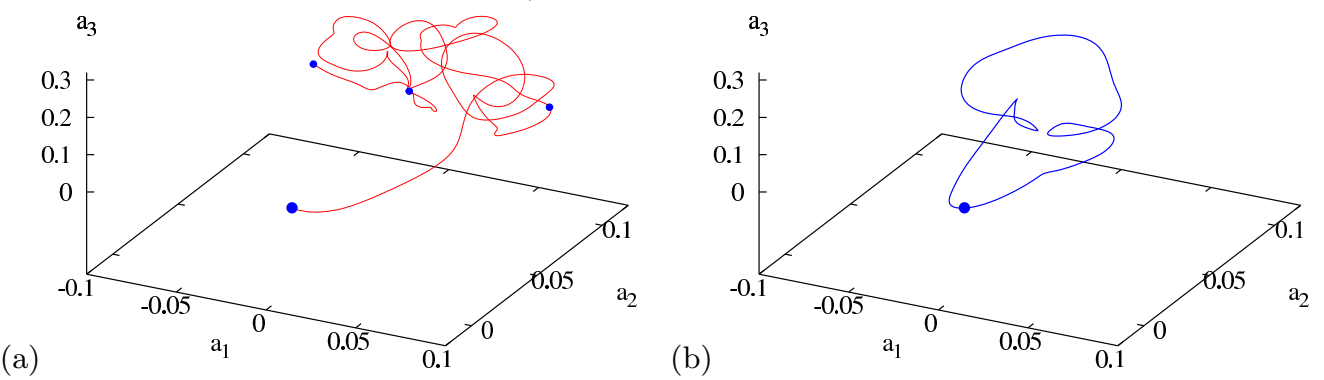

FiguRE 1. Symmetry reduction replaces each full state space trajectory $a(t)$ by a simpler reduced state space trajectory $\hat{a}(t)$, with continuous group induced drifts quotiented out. Here this is illustrated by the relative periodic orbit $\mathrm{RPO}_{36.72}$ (see figure $9 \mathrm{~b}$ ) (a) traced in the full state space for three $T=36.72$ periods, in the frame moving with the constant axial phase velocity $\bar{c}=1.274$, the average phase velocity of structures estimated from a long simulation; dots are spaced $T$ apart in time; (b) restricted to the symmetry-reduced state space. Both are projected onto the 3 -dimensional frame (2.16). In the full state space a relative periodic orbit traces out quasi-periodically a highly contorted 2-torus; in the reduced state space it closes a periodic orbit in one period $T$.

orbits are of particular importance, as they provide the skeleton underpinning the chaotic dynamics (Cvitanović et al. 2011). In shear flows evidence is emerging that the geometry of the state space near the onset of turbulence is governed by a chaotic saddle, a set of unstable solutions and their heteroclinic connections (Mullin \& Kerswell 2005). The long-term goals of this research program are to develop this vision into a quantitative, predictive description of moderate-Re turbulence, and to use this description to control flows and explain their statistics.

In contrast to plane Couette flow, pipe flow has a non-zero mean axial velocity and cannot sustain equilibria and periodic orbits with both broken translational symmetry and zero phase velocity. Hence in pipes, unstable invariant solutions are generically streamwise travelling solutions. The dynamical importance of invariant solutions is specified by periodic orbit theory, in which the contribution of each solution to any dynamical average over the chaotic component of the flow is quantified by a deterministic weight (Cvitanović et al. 2011). In the presence of continuous symmetries periodic orbit theory extends to weighted sums over relative periodic orbits (Cvitanović 2007). While a large number of unstable travelling waves have been identified in pipe flow (Faisst \& Eckhardt 2003; Wedin \& Kerswell 2004; Pringle \& Kerswell 2007; Pringle et al. 2009), their neighbourhoods are visited for only 10-20\% of the time (Kerswell \& Tutty 2007; Schneider et al. 2007; Willis \& Kerswell 2008), and so it is expected that relative periodic orbits capture most of the natural measure of the turbulent flow. Although a few unstable 'tiny' relative periodic orbits have already been found (Duguet et al. 2008; Mellibovsky \& Eckhardt 2011b), these stem from bifurcations of nearby travelling waves and exhibit only minute deviations about them. More recently, Mellibovsky \& Eckhardt (2011a) have identified a new relative periodic orbit appearing at a global Shilnikov-type bifurcation. All these solutions, however, lie far from turbulent dynamics and hence do not provide information about the structure of the chaotic saddle underlying turbulent flow.

One of the main difficulties in identifying relative periodic orbits embedded in turbulence is that each of them travels downstream with its own mean phase velocity. Therefore there is no single co-moving frame that can simultaneously reduce all relative periodic orbits to periodic orbits and all travelling waves to equilibria. This problem is here addressed by the method of slices (Rowley \& Marsden 2000; Beyn \& Thümmler 2004; Siminos \& Cvitanović 2011; Froehlich \& Cvitanović 2011), in which the group orbit of 
any full-flow structure is represented by a single point, the group orbit's intersection with a fixed co-dimension one hypersurface or 'slice'. Although this is analogous to the way a Poincaré section reduces a continuous time orbit to a sequence of points, it should be stressed that a slice is not a Poincaré section. A slice fixes only the group parameters: a continuous time full space orbit remains a continuous time orbit in the symmetry-reduced state space (see figure 1).

Our goals are two-fold. First, we explain what symmetry reduction is and how it can aid in revealing the geometry of the state space of pipe flow. Second, we demonstrate that this new tool enables us to commence a systematic exploration of the hierarchy of dynamically important invariant solutions of pipe flow. Symmetry reduction is here combined with $3 D$ spatial visualisation of instantaneous velocity fields to elucidate the physical processes underlying the formation of unstable coherent structures. Running concurrently, the $\infty$-dimensional state-space representation (Gibson et al. 2008), enables us to track the unstable manifolds of invariant solutions, the heteroclinic connections between them (Halcrow et al. 2009), and provides us with new insights into the nonlinear state space geometry and dynamics of moderate $R e$ wall-bounded flows. Starting in neighbourhoods of the known travelling waves (Pringle et al. 2009) as initial conditions and then searching for close recurrences (Auerbach et al. 1987; Cvitanović \& Gibson 2010) in the reduced state space yields educated guesses for locations of relative periodic orbits. Applying Newton-Krylov methods to these initial guesses leads to the discovery reported here, the first examples of relative periodic orbits embedded into pipe turbulence (see figure $9 b$ ).

The paper is organised as follows. We review pipe flows, their visualisation, and their symmetries in $\S 2$. The method of slices is described in $\S 3$, and the computation of invariant solutions and their stability eigenvalues and eigenvectors in $\S 4$ and $\S 5.1$. The main advances reported in this paper are the symmetry-reduced state space visualisation of moderate- $R e$ turbulent pipe flow, revealing the unstable manifolds of travelling waves, and the determination of new relative periodic orbits ( $(5.2)$. Outstanding challenges are discussed in $\S 6$. Appendix A contains a classification of invariant solutions according to their symmetries.

\section{Pipe flows}

The flow to be considered is that of an incompressible viscous fluid confined within a pipe of circular cross-section, driven by a constant mass-flux in the axial direction. The Reynolds number is defined as $R e=U D / \nu$, where $U$ is the mean velocity of the flow, $D$ is the pipe diameter, and $\nu$ is the kinematic viscosity. We scale lengths by $D$ and velocities by $U$ in the Navier-Stokes equations for $\boldsymbol{u}$, the deviation from the laminar Hagen-Poiseuille flow equilibrium $\boldsymbol{U}(r)=2\left(1-2 r^{2}\right) \hat{\boldsymbol{z}}$,

$$
\frac{\partial \boldsymbol{u}}{\partial t}+\boldsymbol{U} \cdot \boldsymbol{\nabla} \boldsymbol{u}+\boldsymbol{u} \cdot \boldsymbol{\nabla} \boldsymbol{U}+\boldsymbol{u} \cdot \boldsymbol{\nabla} \boldsymbol{u}=-\nabla p+32 \frac{\beta}{R e} \hat{\mathbf{z}}+\frac{1}{R e} \nabla^{2} \boldsymbol{u}, \quad \nabla \cdot \boldsymbol{u}=0 .
$$

Hereafter all times will be expressed in dimensionless units $D / U$. Note that the dimensionless variable $\beta=\beta(t)$ is the fractional pressure gradient needed to maintain a constant mass-flux, additional to that required to drive the laminar flow. A Reynolds number $R e_{p}$, based on the applied pressure gradient, is given by $R e_{p}=R e(1+\beta)$, whereas the friction Reynolds number is $R e_{\tau}=\sqrt{2 R e_{p}}$. The Navier-Stokes equations are formulated in cylindrical-polar coordinates, where $(r, \theta, z)$ are the radius, azimuthal angle and the stream-wise (axial) positions, respectively. The full fluid velocity field $\boldsymbol{U}(r)+\boldsymbol{u}$ is rep- 
resented by $[u, v, w, p](r, \theta, z)$, with $u, v$ and $w$ respectively the radial, azimuthal and stream-wise velocity components, and $p$ the pressure.

In numerical simulations no-slip boundary conditions are imposed at the walls and the infinite pipe is represented by periodic boundary conditions in the stream-wise $z$ direction. Hence the deviation velocity field $\boldsymbol{u}$ and the deviation pressure in the Navier-Stokes equations (2.1) are expanded in Fourier modes in the axial and azimuthal directions,

$$
\boldsymbol{u}\left(r_{n}, \theta, z\right)=\sum_{|k|<K} \sum_{\left|m^{\prime}\right|<M} \boldsymbol{u}_{n k m^{\prime}} \mathrm{e}^{\mathrm{i}\left(2 \alpha k z+m m^{\prime} \theta\right)},
$$

whereas the finite-difference method is used in the radial direction. The computational cell is

$$
\Omega=[1 / 2,2 \pi / m, \pi / \alpha] \equiv\{(r, \theta, z) \in[0,1 / 2] \times[0,2 \pi / m] \times[0, \pi / \alpha]\},
$$

where $L=\pi / \alpha$ is the length of the pipe. While $m=1$ corresponds to the naturally periodic azimuthal boundary condition, e.g. $m=2$ requires that the velocity field repeats itself twice in $\theta$. This study is conducted at

$$
\begin{aligned}
& R e=2400, m=2, \alpha=1.25 \\
& \Omega=[1 / 2, \pi, \pi / 1.25] \approx[90,283,452] \text { wall units },
\end{aligned}
$$

corresponding to a short $L \simeq 2.5 D$-periodic pipe in the stream-wise direction. Mellibovsky \& Eckhardt $(2011 b, a)$ have also focused on $m=2$ and studied cells with $\alpha \in[1.1,1.85]$. Furthermore, in this paper we restrict the dynamics to the 'shift-andreflect' (2.11) invariant subspace: all invariant solutions and turbulence simulations presented here are restricted to this subspace.

In this computational cell at $R e=2400$ the additional pressure fraction required to support turbulence while keeping constant mass-flux is $\bar{\beta}=0.70$, yielding friction Reynolds number $R e_{\tau}=90.3$. Here one pipe radius $D / 2$ corresponds to about 90 wall units. At this Reynolds number and geometry turbulence is found to be transient, with characteristic lifetimes of order $t \approx 10^{3} \mathrm{D} / U$ before the flow finally relaminarises. It is worth noting that in long pipes without symmetry restrictions such a characteristic lifetime is found at $R e=1880$ (Hof et al. 2008; Avila et al. 2010), where the flow takes the form of stream-wise localised puffs.

The domain size (2.4) was chosen as a compromise between the computational preference for small domains vs. the need for the pipe to be sufficiently long to accommodate turbulent dynamics. In addition, restricting the largest wavelength is very useful in identifying key coherent structures characterising turbulent dynamics (Hamilton et al. 1995). Although the pipes studied in this paper are short, the three-dimensional states explored here by equilibria and their unstable manifolds are strikingly similar to typical states in longer pipes.

\subsection{State-space visualization of fluid flows}

As long as one is focusing on a single solution of the Navier-Stokes equations, there are many excellent, physically insightful $3 D$ visualisations of the flow: velocity fields on flow sections, isovorticity surfaces, videos of the flow, and so on. But today dozens of exact equilibrium and travelling wave solutions are known for a given turbulent flow, and the number is steadily growing. Furthermore, we are now commencing an exploration of states of turbulent fluids in terms of unstable periodic orbit solutions, whose number grows exponentially as a function of increasing period. How are we to visualise the totality of these solutions in one go?

The answer was given by Hopf (1948). He envisioned the function space of Navier- 
Stokes velocity fields as an infinite-dimensional state space $\mathcal{M}$ in which each instantaneous state of $3 D$ fluid velocity field $\boldsymbol{u}(\mathbf{x})$ is represented as a unique point $a$. In our particular application we can represent $a=\left(\boldsymbol{u}_{n k m}\right)$ as a vector whose elements are the primitive discretization variables (2.2). The $3 D$ velocity field given by $\boldsymbol{u}_{k n m}(t)$, obtained from integration of the Navier-Stokes equations in time, can hence be seen as trajectory $a(t)$ in $\approx 100,000$ dimensional space spanned by the free variables of our numerical discretisation, with the Navier-Stokes equations (2.1) rewritten as

$$
\dot{a}=v(a), \quad a(t)=a(0)+\int_{0}^{t} \mathrm{~d} t^{\prime} v\left(a\left(t^{\prime}\right)\right) .
$$

Here the current state of the fluid $a(t)$ is the time- $t$ forward map of the initial fluid state $a(0)$. In order to quantify whether two fluid states are close to or far from each other, one needs a notion of distance between two points in state space, measured here as

$$
\left\|a-a^{\prime}\right\|^{2}=\left\langle a-a^{\prime} \mid a-a^{\prime}\right\rangle=\frac{1}{V} \int_{\Omega} d \mathbf{x}\left(\boldsymbol{u}-\boldsymbol{u}^{\prime}\right) \cdot\left(\boldsymbol{u}-\boldsymbol{u}^{\prime}\right) .
$$

There is no compelling reason to use this 'energy norm', other than that velocity fields is what is generated in a numerical computation. What norm one actually uses depends very much on the application. For example, in the study of 'optimal perturbations' that move a laminar solution to a turbulent one, both energy (Tempelmann et al. 2010) and dissipation (Lombardi et al. 2011) norms have been used. In our quest for travelling waves and relative periodic orbits (see $\S 5.2$ ) we find it advantageous to use a 'compensatory' norm (4.3) that enhances the weight of cross-stream velocities.

Visualisations of the state-space trajectory (2.5) are by necessity projections onto two or three dimensions. Flow states can be characterised by the instantaneous kinetic energy of their velocity field, $E=\frac{1}{2}\|\boldsymbol{U}+\boldsymbol{u}\|^{2}$, and energy dissipation rate $D=$ $R e^{-1}\|\boldsymbol{\nabla} \times(\boldsymbol{U}+\boldsymbol{u})\|^{2}$. The dissipation rate is balanced by the energy fed into the flow as

$$
\dot{E}=I-D,
$$

where $I=\frac{1}{V} \oint d S[\mathbf{n} \cdot(\boldsymbol{u}+\boldsymbol{U})] p$ is the external power required to maintain constant mass-flux. A physically appealing choice is to monitor the flow in terms of these symmetryinvariant, physical observables $\left(E(t) / E_{\text {lam }}, D(t) / D_{\text {lam }}, I(t) / I_{\text {lam }}\right)$, as in figure 11 . Note that $I(t) / I_{\text {lam }}=1+\beta(t)$. For travelling waves the kinetic energy is constant, so that $D=I$. Such solutions sit on the diagonal in figure $11(a)$, whereas for relative periodic orbits the kinetic energy is time-periodic, with $\bar{D}=\bar{I}$ only for long-time averages. Whilst this is a good check on correctness of numerical data, such projections bunch all invariant solutions and turbulent flow along the energy-balance lines, even though the solutions themselves can be (and often are) very distant from each other. In fact, if two fluid states are clearly separated in such plot, they are also separated in the high-dimensional state space. However, the converse is not true; states of very different topology might have comparable energies, and such plots may obscure some of the most relevant features of the flow. Furthermore, relations such as (2.7) depend on detailed type and geometry of a given problem (Greene \& Kim 1988; Cvitanović et al. 2009), and further physical observables beyond $(E(t), D(t), I(t))$ are difficult to construct.

Recently, Gibson et al. (2008) have shown that with the state space considered as a high-dimensional vector space, the dynamics can be elucidated more profitably by computationally straightforward sets of physical coordinates. First, one identifies several prominent flow states $\boldsymbol{u}_{A}, \boldsymbol{u}_{B}, \ldots$, such as equilibria and their linearised stability eigenvectors, in whose neighbourhoods the turbulent flow spends most of the time. From 
them an orthonormal basis set $\left\{\mathbf{e}_{1}, \mathbf{e}_{2}, \cdots, \mathbf{e}_{n}\right\}$ is constructed by Gram-Schmidt and/or (anti)-symmetrizations. The evolving fluid state $\mathbf{u}(t)$ is then projected onto this basis using the inner product (2.6),

$$
a(t)=\left(a_{1}, a_{2}, \cdots, a_{n}, \cdots\right)(t), \quad a_{n}(t)=\left\langle\boldsymbol{u}(t) \mid \mathbf{e}_{n}\right\rangle .
$$

Finally, low-dimensional projections of the flow can be viewed in any of the $2 D$ planes $\left(a_{m}, a_{n}\right)$ or in $3 D$ perspective views $\left(a_{\ell}, a_{m}, a_{n}\right)$. An example is the figure 1 projection on the 3 -dimensional frame $\left\{\mathbf{e}_{1}, \mathbf{e}_{2}, \mathbf{e}_{3}\right\}$ defined in (2.16).

It is worth emphasising that this method offers a low-dimensional visualisation without dimension reduction or low-dimensional modelling; the dynamics are computed with fullyresolved direct numerical simulations. Although the use of particular travelling waves to define low-dimensional projections (see § 5.1) may appear arbitrary, the choice turns out to be very useful when the turbulent flow is chaperoned by a few invariant solutions and their unstable manifolds, as for example in low Reynolds number plane Couette flow (Gibson et al. 2008). Such visualisations are essential to uncovering the interrelations between invariant solutions, and constructing symbolic dynamics partitions of state space needed for a systematic exploration of turbulent dynamics. This is the key challenge we address here for the case of turbulent pipe flows.

\subsection{Symmetries of pipe flow}

In many physical applications equations such as those of Navier-Stokes retain their form under symmetry transformations. Consider the Navier-Stokes equations in the state space formulation (2.5). A flow $\dot{a}=v(a)$ is said to be $G$-equivariant if the form of evolution equations is left invariant by the set of transformations $g$ that form the group of symmetries of the dynamics $G$,

$$
v(a)=g^{-1} v(g a), \quad \text { for all } g \in G .
$$

On an infinite domain and in the absence of boundary conditions, the Navier-Stokes equations are equivariant under translations, rotations, and $\mathbf{x} \rightarrow-\mathbf{x}, \mathbf{u} \rightarrow-\mathbf{u}$ inversion through the origin (Frisch 1996). In pipe flow the cylindrical wall restricts the rotation symmetry to rotation about the $z$-axis, and translations along it. Let $g(\phi, \ell)$ be the shift operator such that $g(\phi, 0)$ denotes an azimuthal rotation by $\phi$ about the pipe axis, and $g(0, \ell)$ denotes the stream-wise translation by $\ell$; let $\sigma$ denote reflection about the $\theta=0$ azimuthal angle:

$$
\begin{aligned}
g(\phi, \ell)[u, v, w, p](r, \theta, z) & =[u, v, w, p](r, \theta-\phi, z-\ell) \\
\sigma[u, v, w, p](r, \theta, z) & =[u,-v, w, p](r,-\theta, z) .
\end{aligned}
$$

The Navier-Stokes equations for pipe flow are equivariant under these transformations. The symmetry group of stream-wise periodic pipe flow is thus $G=\mathrm{O}(2)_{\theta} \times \mathrm{SO}(2)_{z}=$ $\mathrm{D}_{1} \ltimes \mathrm{SO}(2)_{\theta} \times \mathrm{SO}(2)_{z}$, where $\mathrm{D}_{1}=\{e, \sigma\}$ denotes azimuthal reflection, $\ltimes$ stands for a semi-direct product (in general, reflections and rotations do not commute), and the subscripts $z, \theta$ indicate stream-wise translation and azimuthal rotation respectively. For an assessment of the discrete symmetries in pipe flow see appendix A.

Whilst the flow equations are invariant under $G$, the state of flow typically is not. Only the laminar Hagen-Poiseuille equilibrium is invariant under all of $G$, whereas a generic turbulent state has only the trivial symmetry group $\{e\}$. In this paper we restrict our investigations to dynamics restricted to the 'shift-and-reflect' symmetry subspace (A 3),

$$
S=\left\{e, \sigma g_{z}\right\},
$$

i.e. velocity fields (2.10) that satisfy $[u, v, w, p](r, \theta, z)=[u,-v, w, p](r,-\theta, z-L / 2)$. In 
addition, in some of the simulations (e.g. figure 8) we further impose the 'rotate-andreflect' symmetry

$$
Z_{2}=\left\{e, \sigma g_{\theta}\right\},
$$

which is possessed by the highly symmetric waves found by Pringle et al. (2009). In this case the velocity field also satisfies $[u, v, w, p](r, \theta, z)=[u,-v, w, p](r, \pi / 2-\theta, z)$.

It is worth emphasising that by imposing the symmetry $S$, rotations are prohibited and hence we consider only the simplest example of a continuous group, the stream-wise one-parameter rotation group $\mathrm{SO}(2)_{z}$, omitting the subscript $z$ whenever that leads to no confusion. In the literature (see, e.g. Recke et al. (2011)) such $\mathrm{SO}(2)$ is often referred to as the circle group $S^{1}$.

\subsection{Symmetry-induced coordinate frames}

So far we have not offered any advice as to the choice of basis vectors in constructing state space coordinates (2.8). In this section we show that the presence of a continuous symmetry suggests two natural mutually orthogonal basis vectors, the group action tangent and curvature vectors, suitable to local visualisations of group orbits.

Consider the one-parameter rotation group $\mathrm{SO}(2)$ acting on a smooth periodic function $u(\theta+2 \pi)=u(\theta)$ defined on the domain $\theta \in[0,2 \pi)$, expanded in the Fourier basis

$$
u(\theta)=\sum a_{m} \mathrm{e}^{\mathrm{i} m \theta} .
$$

Here $u$ is real, so $a_{m}=a_{-m}^{*}$. Let us parametrise forward translations by the continuous parameter $\phi, g(\phi) u(\theta)=u(\theta-\phi)$, or, in Fourier space, $g(\phi) a=\operatorname{diag}\left\{\mathrm{e}^{-\mathrm{i} m \phi}\right\} a$. The tangent to the group orbit at point $a$ is then given by the first derivative with respect to the group parameter, and the direction of curvature by the second derivative,

$$
\begin{aligned}
\mathbf{t}(a) & =\lim _{\phi \rightarrow 0}(g(\phi) a-a) / \phi=\operatorname{diag}\{-\mathrm{i} m\} a=\mathbf{T} a, \\
\kappa(a) \mathbf{n}(a) & =\mathbf{T}^{2} a=-\operatorname{diag}\left\{m^{2}\right\} a,
\end{aligned}
$$

where $\mathbf{n}$ is a unit vector normal to the tangent and $1 / \kappa$ is the radius of curvature. The pair of unit vectors

$$
\left\{\mathbf{e}_{n}, \mathbf{e}_{n+1}\right\}=\{\mathbf{t}(a) /\|\mathbf{t}(a)\|, \mathbf{n}(a)\}
$$

forms a local orthogonal Frenet-Serret frame at state space point $a$, and can be useful in constructing the state space basis vector set (2.8). For example, in figure 1 the relative periodic orbit $\mathrm{RPO}_{36.72}$ is projected onto the 3 -dimensional orthogonal frame

$$
\left\{\mathbf{e}_{1}, \mathbf{e}_{2}, \mathbf{e}_{3}\right\}=\left\{\mathbf{t}\left(a^{\prime}\right) /\left\|\mathbf{t}\left(a^{\prime}\right)\right\|, \mathbf{n}\left(a^{\prime}\right),\left(\hat{a}_{d}-a^{\prime}\right)_{\perp} /\left\|\hat{a}_{d}-a^{\prime}\right\|_{\perp}\right\}
$$

where $a^{\prime}=a(0)$ is a point on the relative periodic orbit (such fluid snapshot is called 'template' or 'reference state' in what follows), $\hat{a}_{d}$ is the most distant point from $\hat{a}$ along its symmetry-reduced state space periodic orbit $\hat{a}(t)$, measured in the energy norm (2.6), and $\left(\hat{a}_{d}-a^{\prime}\right)_{\perp}$ is the component of their separation vector, Gram-Schmidt orthogonalised to $\left\{\mathbf{e}_{1}, \mathbf{e}_{2}\right\}$.

In what follows we consider time-dependent group parameters $\phi(t)$, and the associated phase velocity $\dot{\phi}$ along the group tangent evaluated at the state space point $a$ is given by

$$
g^{-1} \dot{g} a=\mathrm{e}^{-\phi \mathbf{T}}\left(\frac{\mathrm{d}}{\mathrm{d} t} \mathrm{e}^{\phi \mathbf{T}}\right) a=\dot{\phi} \cdot \mathbf{t}(a) .
$$

This formula for the phase velocity is known as the 'Cartan derivative'; for $N$-parameter continuous symmetry the dot product is $N$-dimensional, as in (2.18). 


\subsection{Relative invariant solutions}

In systems with continuous symmetries there are important classes of invariant solutions referred to as 'relative' or 'equivariant' (Huygens 1673; Poincaré 1896). In pipe flows one expects to find travelling waves and relative periodic orbits (Rand 1982) associated with the translational and rotational symmetries of the flow. Although these unstable flowinvariant solutions can only be computed numerically, they are 'exact' in the sense that they converge to solutions of the Navier-Stokes equations as the numerical resolution increases.

A relative equilibrium (labelled here TW for travelling wave) is a dynamical orbit whose velocity field (2.5) lies within the group tangent space

$$
v(a)=c \cdot \mathbf{t}(a),
$$

with a constant phase velocity $\left(\dot{\phi}_{1}, \cdots, \dot{\phi}_{N}\right)=\left(c_{1}, \cdots, c_{N}\right)$ and $\dot{\phi}$ defined in (2.17). Here $N$ is the dimension of the continuous symmetry. In pipe flow $N=2$ and $\left\{\phi_{n}\right\}=\{\phi, \ell\}$, corresponding to rotations and translations. For a travelling wave, time evolution is confined to the group orbit

$$
a(t)=g(c t) a(0), \quad a(t) \in \mathcal{M}_{\mathrm{TW}} .
$$

As a travelling wave explores only its group orbit, a travelling wave is not a periodic orbit. Rather, as all states in a group orbit are physically the same state, this is a generalised equilibrium. In pipe flow relative equilibria can propagate in the streamwise direction $z$ (travelling waves), in azimuthal $\theta$ direction (rotating waves), or both. However, in the shift-and-reflect subspace (2.11) considered here, rotations are precluded. In this case only stream-wise travelling waves are permitted, satisfying (2.19)

$$
\boldsymbol{f}(\boldsymbol{u}(0), t)=g(0,-c t) \boldsymbol{u}(t)-\boldsymbol{u}(0)=\mathbf{0},
$$

where $c$ is the stream-wise phase velocity.

A relative periodic orbit $p$ is an orbit in state space $\mathcal{M}$ which exactly recurs

$$
a(t)=g_{p} a\left(t+T_{p}\right), \quad a(t) \in \mathcal{M}_{p}
$$

after a fixed relative period $T$, but shifted by a fixed group action $g$ that maps the endpoint $a(T)$ back into the initial point cycle point $a(0)$. In pipe flow, a relative periodic orbit $p$ is a time-dependent velocity field

$$
\mathbf{u}_{p}(r, \theta, z, t)=\mathbf{u}_{p}\left(r, \theta+\phi_{p}, z+\ell_{p}, t+T_{p}\right)
$$

that recurs after time $T_{p}$, rotated and shifted by $\phi_{p}$ and $\ell_{p}$. In our Newton search for a relative periodic orbit $p$, we seek the zeros of

$$
\boldsymbol{f}(\boldsymbol{u}(0), T, \ell)=g(0,-\ell) \boldsymbol{u}(T)-\boldsymbol{u}(0)=\mathbf{0},
$$

starting with a guess for the initial state of fluid $\boldsymbol{u}$, period $T$, and shift $\ell$.

Continuous symmetry parameters ('phases' or 'shifts') $\left\{\phi_{n}\right\}=\left\{\phi_{p}, \ell_{p}\right\}$ are real numbers, so ratios $\pi / \phi_{n}$ are almost never rational, and relative periodic orbits are almost never periodic. In pipe flow the time evolution of a relative periodic orbit sweeps out quasi-periodically the 3 -dimensional group orbit $\mathcal{M}_{p}$ without ever closing into a periodic orbit.

\section{Reduction of continuous symmetry}

We have seen that in presence of the continuous $\mathrm{SO}(2)$ symmetry, travelling waves and relative periodic orbits are 2 - and 3 -dimensional manifolds of physically equivalent states 
(a)
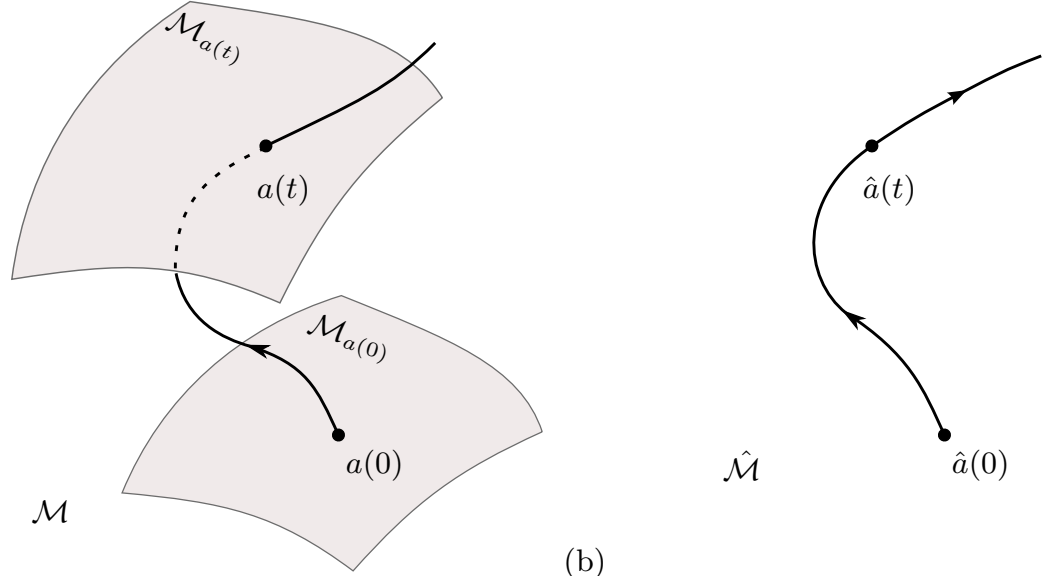

(b)

Figure 2. (a) The group orbit $\mathcal{M}_{a(0)}$ of state space point $a(0)$, and the group orbit $\mathcal{M}_{a(t)}$ reached by the trajectory $a(t)$ time $t$ later. (b) Symmetry reduction $\mathcal{M} \rightarrow \hat{\mathcal{M}}$ replaces each full state space group orbit $\mathcal{M}_{a} \subset \mathcal{M}$ by a single point in the reduced state space $\hat{a} \in \hat{\mathcal{M}}$.

generated by axial and azimuthal shifts. How are we to compare a pair of such states? We start by determining the minimal distance between them.

The group orbit $\mathcal{M}_{a}$ of a state space point $a \in \mathcal{M}$ is traced out by the set of all group actions

$$
\mathcal{M}_{a}=\{g a \mid g \in G\} .
$$

Any state in the group orbit set $\mathcal{M}_{a}$ is physically equivalent to any other. The action of a symmetry group thus foliates the state space into a union of group orbits, figure $2(a)$.

For the example at hand, a pipe flow (or a plane Couette flow) with two periodic boundary conditions, the symmetry group $\Gamma$ contains two commuting $\mathrm{SO}(2)$ rotations. Each $\mathrm{SO}(2)$ subgroup group orbit is (topologically) a circle, see figure 3, and together they sweep out a $T^{2}$ torus, see figure 4 .

The goal of symmetry reduction is to replace each group orbit by a unique point in a lower-dimensional symmetry-reduced state space $\hat{\mathcal{M}}=\mathcal{M} / G$, as sketched in figure 2 . Several symmetry reduction schemes are reviewed in Siminos \& Cvitanović (2011). Here we shall describe the method of slices (Rowley \& Marsden 2000; Beyn \& Thümmler 2004; Froehlich \& Cvitanović 2011), the only method that we find practical for a symmetry reduction of turbulent solutions of highly nonlinear flows, see $\S 5.2$.

In the method of slices the symmetry reduction is achieved by cutting the group orbits with a finite set of hyperplanes, one for each continuous group parameter, with each group orbit of symmetry-equivalent points represented by a single point, its intersection with the slice. The procedure is akin to (but distinct from) cutting across continuoustime parametrised trajectories by means of Poincaré sections. As is the case for Poincaré sections, choosing a 'good' slice is a dark art. Our guiding principle is to chose a slice such that the distance between a 'template' state $a^{\prime}$ and nearby group orbits is minimised, i.e., identify the point $\hat{a}$ on the group orbit (3.1) of a nearby state $a$ which is the closest match to the template point $a^{\prime}$.

\subsection{Method of slices; local charts}

After some experimentation and observations of turbulence in a given flow, one can identify a set of dynamically important unstable recurrent coherent structures. For example, coherent streaky structures have been observed in pipe flow at transitional $R e$ 

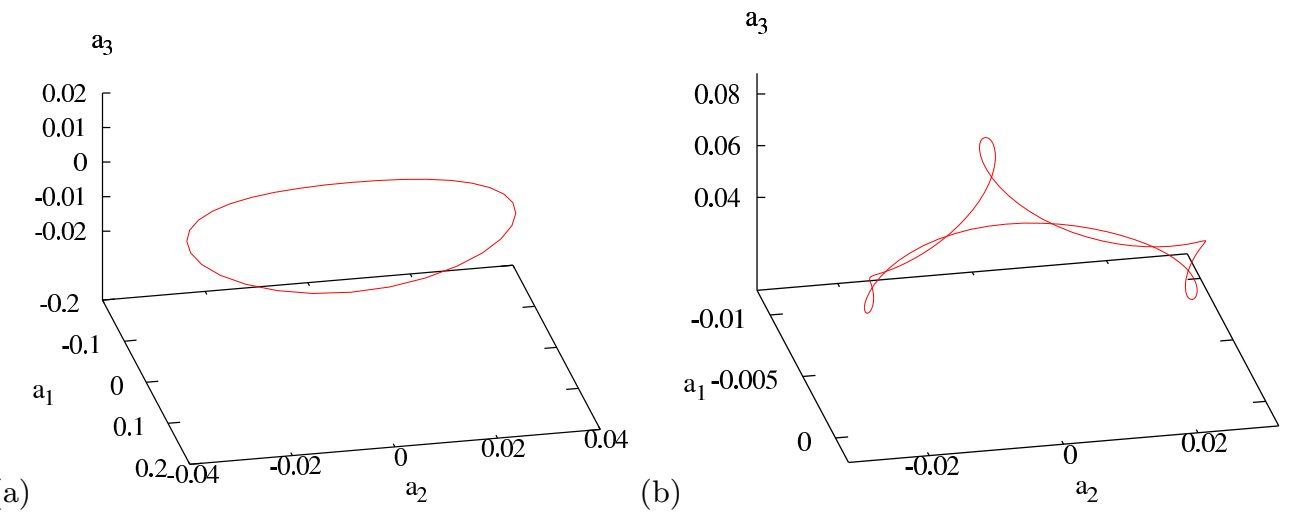

Figure 3. Projections of group orbits of two states $a$ (in $\approx$ 100,000-dimensional state space) onto stationary Frenet-Serret frames given by unit vectors in the directions $\left\{\mathbf{t}_{z}\left(a^{\prime}\right), \mathbf{t}_{\theta}\left(a^{\prime}\right), \mathbf{n}_{z}\left(a^{\prime}\right)\right\}$, see (2.15). The state in (a) is a very smooth state, the 'lower-branch' travelling wave LB, whereas in (b) it is a snapshot from a turbulent run. The group orbits are generated by all possible axial shifts $g(0, \ell) a$, and plotted relative to a template point $a^{\prime}$. In (a) the state $a=a^{\prime}=a_{\mathrm{LB}}(0)$ belongs to the "lower-branch" travelling wave $\mathcal{M}_{\mathrm{LB}}$ described in $\S 5.1$; in (b) $a$ is a 'typical' turbulent state shapshot with its group orbit as seen from the template $a^{\prime}=a_{\mathrm{ML}}$. Group orbits are only topologically circles; for strongly nonlinear, turbulent states many Fourier modes are of comparable magnitude, with their sums resulting in highly convoluted group orbits such as (b).
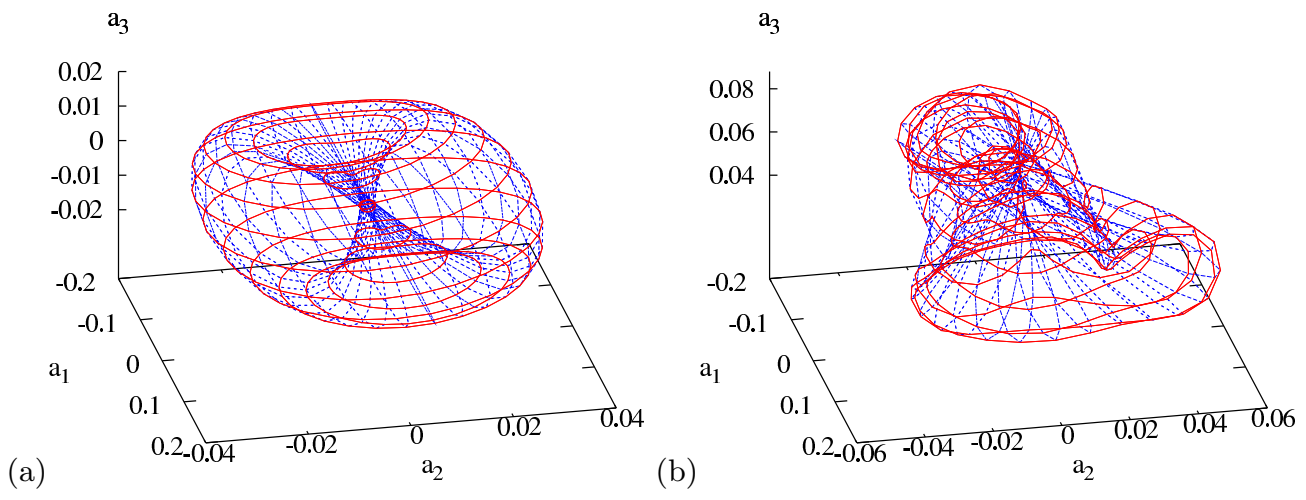

FiguRE 4. As figure 3, but with the full 2-dimensional $\mathrm{SO}(2)_{\theta} \times \mathrm{SO}(2)_{z}$ group orbits traced out by shifts in both $z$ and $\theta$. Loops in solid red correspond to shifts in $z$, dashed blue loops to shifts in $\theta$.

(Hof et al. 2004) through to very high Re (Kim \& Adrian 1999) where 'very large scale motions' have length scales comparable to the pipe radius. Streaky structures are also observed in the buffer layer of turbulent flows with a characteristic span-wise wavelength of approximately 100 wall units (Kline et al. 1967).

We shall refer to this catalogue of $n$ representative snapshots or 'reference states', either precomputed or experimentally measured, as templates (Rowley \& Marsden 2000), each an instantaneous state of the $3 D$ fluid flow represented by a point $a^{\prime(j)}, j=1,2, \cdots, n$, in the state space $\mathcal{M}$ of the system. Symmetries of the flow (i.e. the $g \in G$ ) are then used to shift and rotate the template $a^{\prime}$ until it overlies, as well as possible, the coherent structure of interest $a$, by minimising the distance

$$
\left\|a-g(\phi) a^{\prime}\right\| .
$$

The entire group orbit of $a$ is then replaced by the closest match to the template pattern, 
(a)
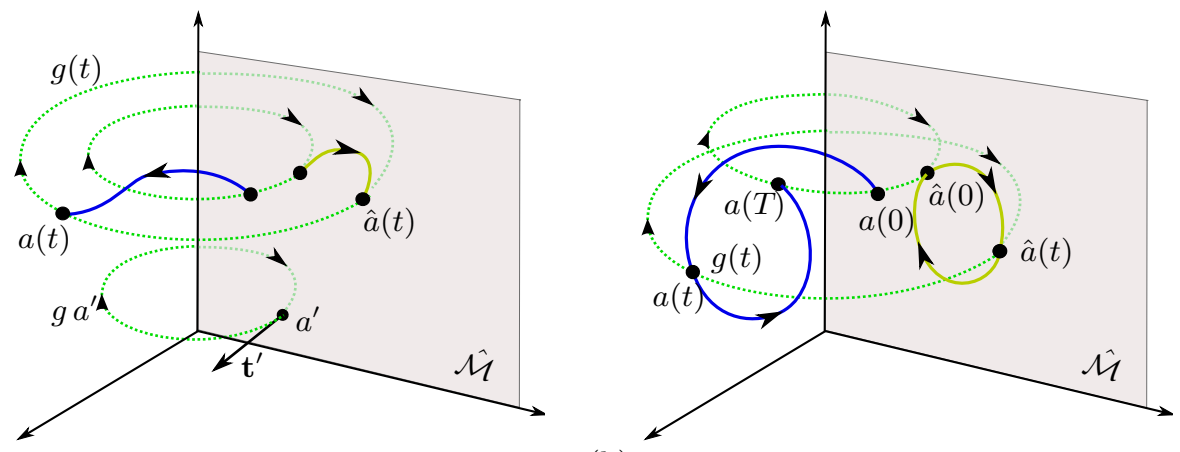

(b)

FiguRE 5. The method of slices, a state space visualisation: (a) Slice $\hat{\mathcal{M}} \supset \mathcal{M} / G$ lies in the $(d-N)$-dimensional hyperplane (3.3) normal to $\mathbf{t}^{\prime}$, where $\mathbf{t}_{j}^{\prime}$ span the $N$-dimensional space tangent to the group orbit $g a^{\prime}$ (dotted line) evaluated at the template point $a^{\prime}$. The hyperplane intersects all full state space group orbits (green dashes). The full state space trajectory $a(t)$ (blue) and the reduced state space trajectory $\hat{a}(t)$ (green) are equivalent up to a 'moving frame' rotation $a(t)=g(t) \hat{a}(t)$, where $g(t)$ is a shorthand for $g(\phi(t))$. (b) In the full state space $\mathcal{M}$ a relative periodic orbit $a(0) \rightarrow a(t) \rightarrow a(T)$ returns to the group orbit of $a(0)$ after time $T$ and a rotation by $g, a(0)=g a(T)$. For flows with continuous symmetry a generic relative periodic orbit fills out quasi-periodically what is topologically a torus. In the slice $\hat{\mathcal{M}}$ the symmetry-reduced orbit is periodic, $\hat{a}(0)=\hat{a}(T)$. This is a highly idealised sketch: A group orbit is a $N$-dimensional manifold, and even for $\mathrm{SO}(2)$ it is usually only topologically a circle (see figure 3), and can intersect a hyperplane any number of times (see figure $6 a$ ).

given by $\hat{a}=g^{-1} a$, as shifting does not affect the norm, $\left\|a-g a^{\prime}\right\|=\left\|\hat{a}-a^{\prime}\right\|$. The symmetry-reduced state space $\hat{\mathcal{M}}$ (hereafter referred to as the 'slice'), of dimension $(d-1)$, consists of the set of closest matches $\hat{a}$, one element for each full state space $\mathcal{M}$ group orbit; the hat on $\hat{a}$ indicates the unique point on the group orbit of $a$ closest to the template $a^{\prime}$.

For the azimuthal $\mathrm{SO}(2)_{\theta}$ rotations (and likewise for the periodic pipe $\mathrm{SO}(2)_{z}$ streamwise translations), the minimal distance satisfies the extremum condition

$$
\frac{\partial}{\partial \phi}\left\|a-g(\phi) a^{\prime}\right\|^{2}=2\left\langle a-g a^{\prime} \mid \mathbf{T}_{\theta} g a^{\prime}\right\rangle=2\left\langle\hat{a}-a^{\prime} \mid \mathbf{T}_{\theta} a^{\prime}\right\rangle=0,
$$

given that group orbits are smooth differentiable manifolds. As $\left\|g(\phi) a^{\prime}\right\|$ is a constant, the group tangent vector $\mathbf{T}_{\theta} a^{\prime}$ evaluated at $a^{\prime}$ (2.13) is normal to $a^{\prime}$, and the term $\left\langle a^{\prime} \mid \mathbf{T}_{\theta} a^{\prime}\right\rangle$ vanishes $\left(\mathbf{T}_{\theta}\right.$ is antisymmetric). Therefore the point $\hat{a}$ on the group orbit that lands in the slice, satisfies the slice condition

$$
\left\langle\hat{a} \mid \mathbf{t}_{\theta}^{\prime}\right\rangle=0, \quad \mathbf{t}_{\theta}^{\prime}=\mathbf{T}_{\theta} a^{\prime} .
$$

The slice so defined is thus a hyperplane that includes the origin, normal to the template group tangent evaluated at the template.

When $a$ is varies in time, $\dot{a}=v(a)$, the template $a^{\prime}$ tracks the motion using the slice condition (3.3) to minimise $\left\|a(t)-g(\phi(t)) a^{\prime}\right\|$, and the full-space trajectory $a(t)$ is thus rotated into the reduced state space, $\hat{a}(t)=g^{-1} a(t)$, by appropriate moving frame (Cartan 1935; Fels \& Olver 1998, 1999; Olver 1999) angles $\left\{\phi(t)_{n}\right\}$, as depicted in figure $5(a)$. Specializing to $\mathrm{SO}(2)$, one can write the equations for the reduced state space flow, $\hat{a}(t) \in \hat{\mathcal{M}}$ confined to the slice, $\dot{\hat{a}}=\hat{v}(\hat{a})$, as

$$
\begin{aligned}
\hat{v}(\hat{a}) & =v(\hat{a})-\dot{\phi}(\hat{a}) \mathbf{t}(\hat{a}) \\
\dot{\phi}(\hat{a}) & =\left\langle v(\hat{a}) \mid \mathbf{t}^{\prime}\right\rangle /\left\langle\mathbf{t}(\hat{a}) \mid \mathbf{t}^{\prime}\right\rangle .
\end{aligned}
$$


(a)
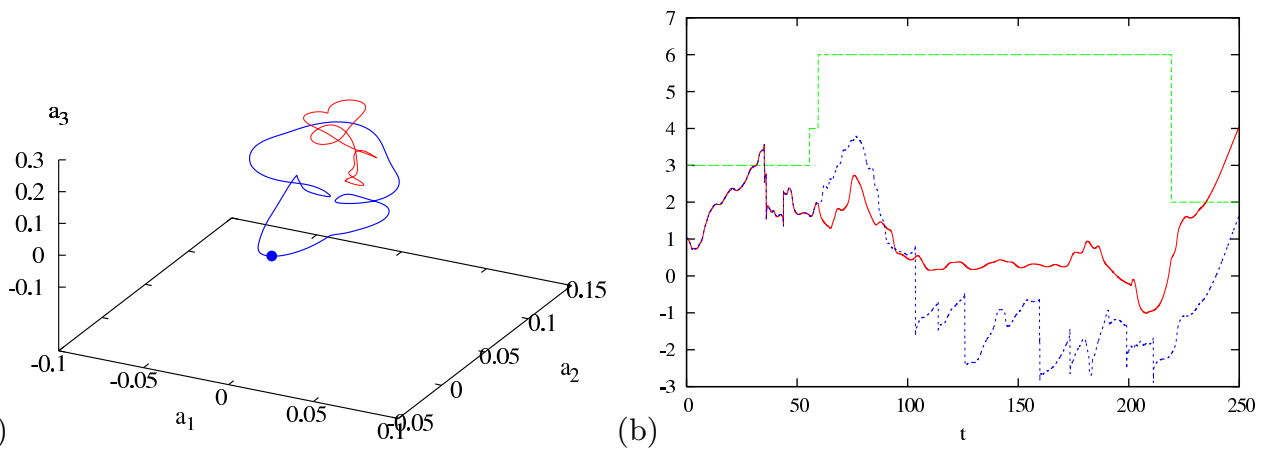

FiguRE 6. (a) Every slice hyperplane cuts every group orbit at least twice (see figure 5). An $\mathrm{SO}(2)$ relative periodic orbit is topologically a torus, so the two cuts are the two periodic orbit images of the same relative periodic orbit, the good close one, and the bad distant one, on the other side of slice border, and thus not in the slice. Here this is illustrated by close cut (blue, solid line) of the relative periodic orbit $\mathrm{RPO}_{36.72}$ torus, figure $1(b)$, plotted together with the most distant cut (red, dashed), in the same slice hyperplane, but not in the slice. (b) Comparison of symmetry-reduced trajectory using a single template ML (blue, short-dash) with the same trajectory symmetry reduced using the travelling waves in table 1 as template (red, solid) indexed by $j(t)(j=1$ laminar; $2 \mathrm{LB} ; 3 \mathrm{ML} ; 4 \mathrm{MU} ; 5 \mathrm{UB} ; 6 \mathrm{~S} 2 \mathrm{U} ; 7 \mathrm{~S} 2 \mathrm{~L})$, with template at time $t$ indicated on left ordinate by (green, long-dash) line. Right ordinate: the shift deviation from the mean shift, $\ell(t)-\bar{c} t$, where $\bar{c} \simeq 1.274$ is estimated by a long-time simulation. Both symmetry reductions begin with the same template and experience the same jumps in the shift starting at $t \approx 40$. By starting to switch the templates at $t \approx 60$, further jumps (seen for the blue, short-dash line) are avoided (red, solid line).

In other words, $v$, the velocity in the full state space, can be written as the sum of $\hat{v}$, the velocity component in the slice, and $\dot{\phi} \mathbf{t}$, the Cartan derivative (2.17) or the velocity component along the group tangent directions. The $\dot{\phi}$ equation is the reconstruction equation: its integral keeps track of the group shifts in the full state space. In particular, if $\hat{a}$ is a point on a travelling wave (2.18), the full state space velocity equals the phase velocity, and $\hat{v}(\hat{a})=0$, i.e., travelling waves are always reduced to equilibria in the slice. It should be emphasised that we never integrate the reduced equations (3.4); numerical simulations are always carried out in the full state space. Slicing is implemented as postprocessing of numerical or experimental data, by rotating full state space trajectories into the slice, as in figure 5 .

\subsection{Charting the reduced state space; a global atlas}

The method of slices as implemented here associates a slice (3.3) to a template. Our slice is locally a hyperplane, expected to be a good description of solutions similar to a given template only in its neighbourhood. Nevertheless, as every group orbit has a point closest to a given template, and a slice is the set of all such group-orbit points, it slices the group orbits of all full state space points. The variational distance condition (3.3) is an extremum condition, and as the group orbits of highly nonlinear states are highly contorted (see figure $4 b$ ), the distance function can have many extrema, and multiple sections by a slice hyperplane. For example, a relative periodic orbit sweeps out a torus, and is always intersected by a slice hyperplane in two or more periodic orbit sections, once at the orbit's closest passage to the template, with positive curvature (2.14), and another time at the most distant passage, also satisfying the slice condition (4.2), but with negative curvature (see figure $6 a$ ).

As explained in Froehlich \& Cvitanović (2011), a slice hyperplane captures faithfully neighboring group orbits as long as it slices them well; it does so until it reaches the 
chart border, the set of points $\hat{a}^{*}$ sufficiently far from the template, whose group orbits are grazed tangentially rather than sliced transversely. For such grazing group orbit the group tangent vector lies in the slice, and is thus orthogonal to the slice tangent,

$$
\left\langle\mathbf{t}\left(\hat{a}^{*}\right) \mid \mathbf{t}^{\prime}\right\rangle=0 .
$$

The phase velocity $\dot{\phi}\left(\hat{a}^{*}\right)$ in (3.5) then diverges. While such divergence is an avoidable nuisance, an artifact of the symmetry reduction to a particular slice hyperplane, it is a numerical nuisance nevertheless.

For points beyond the chart border (3.6) the group orbits have more than one intersection with the slice. It is clear what the trouble with any single slice hyperplane is: the nonlinear flow of interest is taking place on a highly contorted curved manifold embedded in the $\infty$-dimensional state space, so a single template cannot be a good match globally. It is as good as a projection of the whole Earth onto a single flat map centered on Ulan Bator. The physical task is to, in order to chart the state space of a turbulent flow, pick a set of qualitatively distinct templates $a^{\prime(j)}$ whose slices $\mathcal{M}^{(j)}$ span across neighbourhoods of the qualitatively most important coherent structures, and which together capture all of the asymptotic dynamics and provide a global atlas of the dimensionally reduced state space $\hat{\mathcal{M}}=\mathcal{M} / G$. The choice of templates should reflect the dynamically dominant patterns seen in the solutions of nonlinear PDEs, one typical of, let us say, 2-roll states, one for 4-roll states, and so on. Each slice hyperplane comes with its chart border hyperplane of points $\hat{a}^{*}$, defined by the grazing condition (3.6), beyond which it should not be deployed as a chart. Together they 'Voronoi' tessellate the curved manifold in which the symmetry-reduced strange attractor is embedded by a finite set of hyperplane tiles.

For example, in reducing turbulent trajectories of $\S 5.2$, we deploy a set of travelling waves as our templates. Each associated slice $\mathcal{M}^{(j)}$, provides a local chart at $a^{\prime(j)}$ for a neighbourhood of an important, qualitatively distinct class of solutions. In our simulations we keep checking the distance to the template of the symmetry-reduced trajectory, and switch to the next template neighbourhood before the trajectory encounters the chart border (3.6) of the current one, as illustrated by figure $6(b)$.

\section{How to slice a pipe}

Slicing is independent of numerical representation. We describe our implementation, however, using the convenient discretisation for pipe flow of (2.2). The deviation velocity field $\boldsymbol{u}$ and deviation pressure in the Navier-Stokes equations (2.1) are discretised as in (2.2), using Fourier modes in the axial and azimuthal directions and a finite-differences in the radial direction, with coefficients $\boldsymbol{u}_{n k m}$. The radial points, $r_{n}$ for $n=1,2, \cdots, N$, are non-uniformly spaced, with higher resolution towards the wall. Flow variables being real implies that the coefficients satisfy $\boldsymbol{u}_{n k m}=\boldsymbol{u}_{n,-k,-m}^{*}$. Time-stepping has been performed using a second-order predictor-corrector method with a time-step of $\Delta t=0.0025$. To ensure dealiasing in the evaluation of nonlinear terms, Fourier series are evaluated on $3 K$ and $3 M$ spatial points in $z$ and $\theta$ respectively. For the calculations presented, a resolution of $(N, K, M)=(64,16,16)$ has been used, corresponding to $64 \times 48 \times 48$ grid points.

\subsection{Rotation into the slice}

In this paper we consider only shifts $\ell$ in the stream-wise direction (2.10). Denoting our state by $a=\left(\boldsymbol{u}_{n k m}\right)$, the group tangent $\mathbf{t}(a)=\mathbf{T}_{z} a$ to $a$ in the direction of axial shifts is given by $(2.13)$,

$$
\mathbf{t}(\boldsymbol{u})_{n k m}=-2 \alpha k \text { i } \boldsymbol{u}_{n k m}
$$


and the shift $\ell(t)$ of state space trajectory $a(t)$ into the slice is determined by the slice condition (3.3),

$$
f(\ell(t))=\left\langle g(0,-\ell(t)) a(t) \mid \mathbf{t}^{\prime}\right\rangle=0,
$$

where $\mathbf{t}^{\prime}$ is the group orbit tangent evaluated at a template state $a^{\prime}$. As long as the norm is discretisation independent, the slice condition (4.2) is independent of the numerical representation of the flow $\boldsymbol{u}$, be it finite difference, spectral, and so on. The slice condition is solved for $\ell(t)$ every few time steps using Newton's method, where a good initial guess for $\ell(t)$ is obtained from the previous value and $\dot{\ell}(t)$.

When $\hat{a}(t)$ is close to $a^{\prime}$, the function $f(\ell)$ has only one root. When $a(t)$ is far from $a^{\prime}$, however, $f(\ell)$ may have many roots, pairs of which may disappear with time. This would lead to a discontinuity in $\ell(t)$. As explained in $\S 3.2$, in order to avoid this, a global atlas has to be pieced together from local slice charts, fixed by a well-chosen set of templates $a^{\prime(j)}$. Shifts $\ell_{j}(t)$ are tracked for each local slice chart $\mathcal{M}^{(j)}$, and the next slice hyperplane $\mathcal{M}^{(j+1)}$ with $\ell_{j+1}(t)$ is selected whenever the distance to the next template minimises $\left\|\hat{a}(t)-a^{\prime(j+1)}\right\|$.

\subsection{Dynamically important solutions and Newton's method}

For pipe flows many stream-wise travelling waves satisfying (2.20) are known, and can be used as the starting points for our relative periodic orbit searches. The most of known solutions have no azimuthal precession. This is usually imposed by symmetry, but one could argue that it is the strong stream-wise advection that favours structures with very weak azimuthal rotation speed, empirically $\left|c_{\theta}\right| \leq O\left(10^{-3}\right)$. Stream-wise travelling waves evolve in time along their group orbit, generated by $g(0, \ell(t))$. They therefore satisfy the slice condition (4.2) for $\ell(t)=\ell_{0}+c_{z} t$.

The few pipe flow relative periodic orbits that have been found prior to this study were located via tracking a Hopf bifurcation off a travelling wave solution (Duguet et al. 2008; Mellibovsky \& Eckhardt 2011b). These 'modulated travelling waves,' here referred to as 'tiny' travelling waves, stick close to their mother orbits, and explore little of the state space, with temporal dynamics barely distinguishable from parental travelling waves. In contrast, in our Newton searches for relative periodic orbits, we seek the zeros of relative periodic orbit condition (2.22) deep in the turbulent sea. The way in which the method of slices enables one to find initial guesses for $(\boldsymbol{u}(0), T, \ell)$, is the main difference between this study and the previous searches for relative periodic orbits in pipe flows.

Here we take as initial guesses samples of nearly recurrent velocity fields generated by long-time simulations of turbulent dynamics (Auerbach et al. 1987; Cvitanović \& Gibson 2010). The intent is to find the dynamically most important solutions, by sampling the turbulent flow's natural measure. In practice, sufficiently good full state space initial guesses for $(\boldsymbol{u}(0), T, \ell)$ would be almost impossible to find. Checking correlations between $\boldsymbol{u}(t)$ and $g(0, \ell) \boldsymbol{u}(t-T)$ for each $T$, and more problematically, for all possible shifts $(\phi, \ell)$, is an unrealistic task. The method of slices, however, enables us to determine close recurrences from the symmetry-reduced time series, and locates the dynamically most important solutions, i.e., those trajectories that are most likely to be observed in a longtime turbulent simulation. The relative periodic orbits are reduced to periodic orbits, whose unstable manifolds are much easier to track in the reduced state space. The relative periodic orbit shift $\ell$ is given by the reconstruction equation, (3.5), or, in practice, by phase shift $\ell(T)-\ell(0)$, where $\ell(t)$ is quickly calculated by intermediate Newton steps.

With a good initial guess for $(\boldsymbol{u}(0), T, \ell)$, such a system can be solved using a Newton scheme. Two conditions in addition to (2.22) need to be enforced: the Newton update should have no component along the group orbit, $\langle\boldsymbol{\delta} \boldsymbol{u} \mid \mathbf{t}(\boldsymbol{u})\rangle=0$, and no component 
tangent to trajectory, $\langle\boldsymbol{\delta} \boldsymbol{u} \mid \boldsymbol{\boldsymbol { u }}\rangle=0$. To solve this system a 'hookstep' trust-region variation to the Newton-Krylov method has been implemented, similar to that of Viswanath (2007). This method greatly increases the tolerance in the starting $(\boldsymbol{u}(0), T, \ell)$ required for convergence to an exact solution.

The radial and azimuthal components of the flow are typically smaller than the streamwise component by a factor of approximately 3 to 10 . The components $u$ and $v$, however, can be associated with 'rolls' in the flow that are as important to the self-sustaining mechanism of turbulence as 'streaks', associated with deviations in the $w$ component. This observation motivates use of an empirical 'compensatory norm',

$$
\|\boldsymbol{u}\|_{c}^{2}=\langle\boldsymbol{u} \mid \boldsymbol{u}\rangle_{c}=\frac{1}{2} \int_{V}(9 u \cdot u+9 v \cdot v+w \cdot w) \mathrm{d} V,
$$

found to be useful for the calculation of recurrences and to assist convergence in our Newton scheme.

\section{The sliced pipe}

For our first exploration of the state space of pipe flow, we have chosen a cell size and Reynolds number combination (2.4) empirically balanced so that $R e$ is just sufficient to sustain long periods of turbulence. Among the many travelling waves already known, we have chosen to focus on the family of solutions classed N2 in Pringle et al. (2009). At the parameter values (2.4), the N2 family has the upper and lower branches (UB and LB), as well as two middle states (MU and ML), where 'upper' and 'lower' refers to the friction or dissipation (2.7), associated with each state. The middle states should not be confused with the M branch of solutions documented in Pringle et al. (2009), for which the M2 branch does not appear to exhibit solutions at these particular parameters. States of the $\mathrm{S}$ class do exist at these parameter values, however. Figure 7 shows all travelling waves considered in this work.

\subsection{Sliced travelling waves}

For the sake of simplicity we consider first the dynamics restricted to the $\left(S, Z_{2}\right)$ symmetry subspace of the N2 states. A convenient property of the ML state at our parameter values (2.4) is that it has only one complex unstable eigenvalue within this symmetry subspace. The trajectories of small perturbations therefore spiral away from ML as they follow its unstable manifold. With ML drifting in the axial direction, this local spiral would be difficult or impossible to detect. Within the slice, however, the ML state is reduced to an equilibrium and the local spiral structure is clear, as shown in figure $8(a)$. To project onto the two dimensions of the page, deviations from the ML state have been projected as in (2.8), against the real and imaginary components of its complex stability eigenvector, $\hat{\mathbf{e}}_{1}$ and $\hat{\mathbf{e}}_{2}$ respectively,

$$
\hat{a}_{i}(t)=\left\langle\hat{a}(t)-\hat{a}_{\mathrm{ML}} \mid \hat{\mathbf{e}}_{i}\right\rangle .
$$

Once trajectories escape the neighbourhood of ML they are attracted to another state space region where the UB solution is to be found. Applying the same projection to all N2 travelling waves, figure $8(b)$ shows trajectories along their most unstable directions. Shooting in opposite directions along the most unstable, 1-dimensional manifold of the MU state, one direction goes directly towards the UB state, the other spirals around the ML state first. All trajectories within the $\left(S, Z_{2}\right)$ symmetry subspace are attracted towards a region close to the UB state, where the dynamics is mildly chaotic. In this 


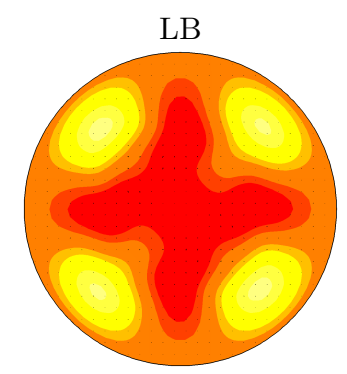

UB

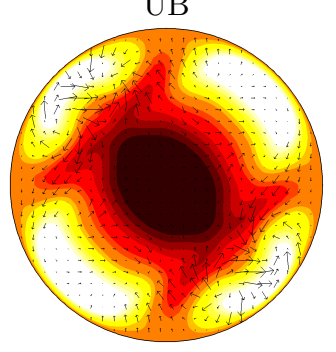

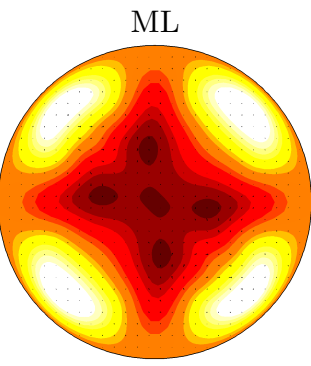

$\mathrm{S} 2 \mathrm{U}$

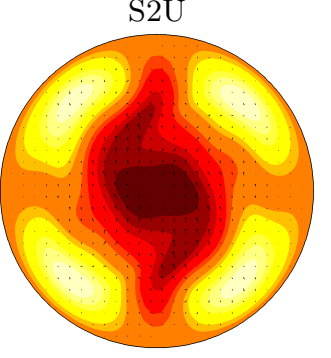

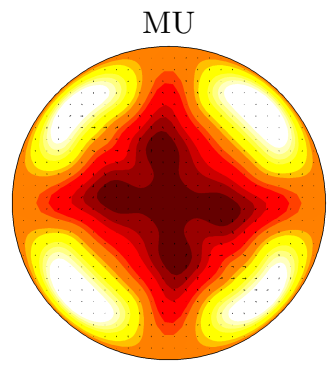

$\mathrm{S} 2 \mathrm{~L}$

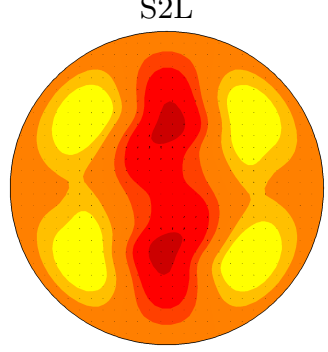

Figure 7. Travelling waves for the cell (2.4), reduced to equilibria by the method of slices. Colour map of stream-wise velocity relative to the laminar flow, lighter (darker) indicating positive (negative) $w$ in the range $[-0.6,0.7]$. The N2 states (LB, ML, MU and UB) have symmetries $\left(S, Z_{2}\right)$, where the symmetry $\mathrm{C}_{2, \theta}$ is implied. The $\mathrm{S} 2$ states originate from a symmetry-breaking bifurcation off the N2 branch and have symmetries $S$ and $\mathrm{C}_{2, \theta}$ only. Shown is one fixed pipe section for each of the solutions. As the choice of the stream-wise position of such section is arbitrary, only meaningful comparison of different solutions is by their distance in the symmetry-reduced state space.

(a)

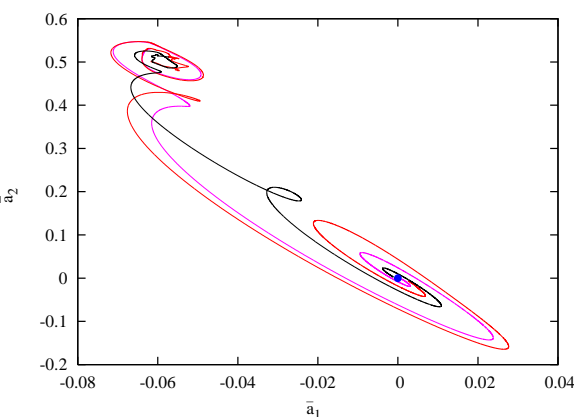

(b)

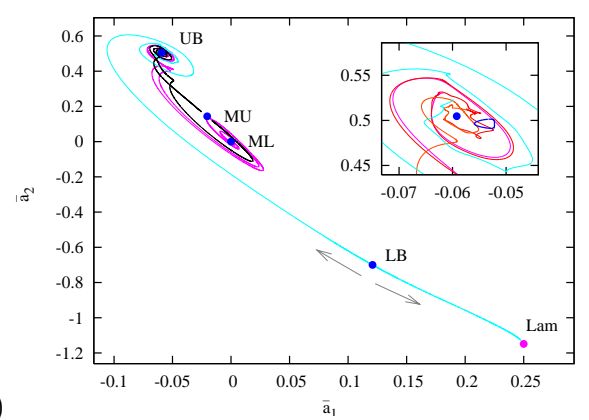

Figure 8. (a) Projection of the dynamics local to the ML travelling wave which has been reduced to the equilibrium at $(0,0)$ within the slice. The local spiral of unstable trajectories is now clearly revealed, the ML state having only a single complex unstable eigenvalue within its $\left(S, Z_{2}\right)$ symmetry subspace. (b) All N2 equilibria, perturbations restricted to the $\left(S, Z_{2}\right)$ symmetry subspace. The inset shows an expanded view near the UB state. The dark blue loop is a tiny relative periodic orbit with period $T=4.934$. Axes as in (5.1).

region we have found a weakly unstable tiny relative periodic orbit of period $T=4.934$, shown in the inset to figure $8(b)$, that appears to dominate the long-time dynamics within the $\left(S, Z_{2}\right)$-invariant subspace. Shooting in opposite directions from the LB state, trajectories proceed directly to either the upper region or the laminar state, suggesting that LB travelling wave lies within the laminar-turbulent boundary or 'edge'.

Without restriction of dynamics to the $\left(S, Z_{2}\right)$-invariant subspace, trajectories stray much further from ML and show turbulent behavior. In order to track such trajectories all 
(a)

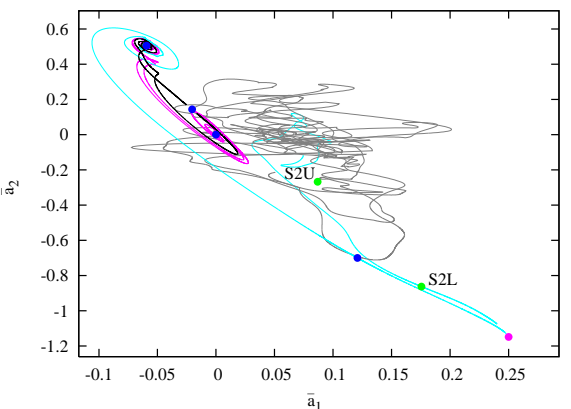

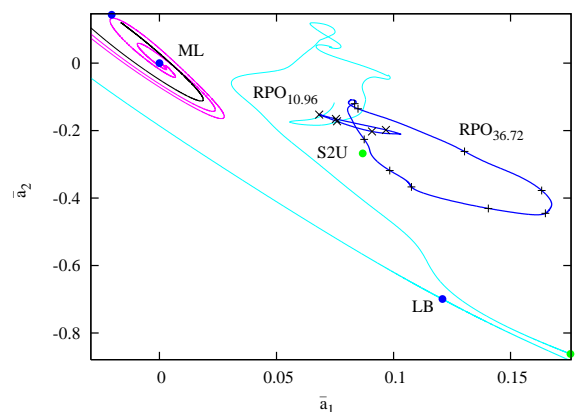

Figure 9. (a) Removal of the $Z_{2}$ symmetry of figure 8 opens the system to far more chaotic, or 'turbulent', transients (grey) within the $S$ symmetry solution space that appear to frequently visit the travelling wave $\mathrm{S} 2 \mathrm{U}$, while the travelling wave $\mathrm{S} 2 \mathrm{~L}$ appears embedded into the laminarturbulent boundary. The turbulent trajectory exhibits excursions to other states as well, most frequently ML and LB. (b) Two relative periodic orbits embedded within turbulence, the same symmetry-reduced state space projection as figure 8 . Crosses are spaced every $\Delta t=2 D / U$ on relative periodic orbit $\mathrm{RPO}_{10.96}$ and pluses are spaced $4 \mathrm{D} / U$ on relative periodic orbit $\mathrm{RPO}_{36.72}$. On this scale the tiny $\mathrm{RPO}_{4.934}$ [figure $8(b)$, inset] only explores a region about the size of the plot dots, and plays no role in turbulent dynamics.

travelling waves states listed in table 1 were deployed as templates, $a^{\prime(j)}, j=1,2, \cdots, 6$, whereas the single template point $a^{\prime}=a_{\mathrm{ML}}(0)$ sufficed for the symmetry reductions within the $\left(S, Z_{2}\right)$ subspace. Switching from one local slice to the next nearest one keeps the phase velocity (3.5) finite (see figure $6 b$ ) and enables tracking of turbulent trajectories in the reduced state space.

A typical trajectory is shown in figure $9(a)$. Within the $\left(S, Z_{2}\right)$-invariant subspace trajectories hover near the UB state; but when $Z_{2}$ symmetry is relaxed, and only $S$ symmetry is enforced, the trajectories explore a far greater region of state space, and appear to be representative of turbulence in the full state space. The neighbourhood of the S2U state is visited frequently, and excursions to other states are occasionally seen. Interestingly, an excursion is observed towards the LB state. Its attracting manifold therefore appears to penetrate into the turbulent region, and, as it lies on the laminar turbulent boundary, attraction towards this manifold may be responsible for the observed sudden relaminarisation events.

\subsection{Relative periodic orbits in pipe flow}

Without symmetry reduction, the detection of a recurrence, i.e. that current state is close in structure to an earlier state on the same trajectory, requires calculating the minimum distance between their group orbits, i.e., minimum over all possible shifts. Within the symmetry-reduced state space the determination of recurrences is simple by construction, a slice is the set of all nearby group orbit states closest to a given template, with symmetry shifts quotiented out, hence all group orbits are reduced to points, and all relative periodic orbits to periodic orbits. The shifts $\ell_{p}$ are determined by the slice condition (4.2). Figure 10 shows a recurrence plot used to detect the signal of a turbulent trajectory that shadows a nearby relative periodic orbit. The indicated minimum at $\Delta t \approx 11$ and its repeats are seen for a while as the relative periodic orbit $\mathrm{RPO}_{10.96}$ is shadowed for a rather long time. States from this minimum, along with the relative stream-wise shift for the candidate trajectory, $\ell(t)-\ell(t-\Delta t)$, were passed to our Newton-Krylov code. This led to the discovery of the relative periodic orbit that we label $\mathrm{RPO}_{10.96}$, and another recurrence plot led to the relative periodic orbit $\mathrm{RPO}_{36.72}$, both 


\begin{tabular}{|c|c|c|c|c|c|c|c|c|}
\hline & & $\frac{\bar{E}}{E_{\text {lam }}}$ & $\frac{\bar{D}}{D_{\operatorname{lam}}}$ & $\bar{c}$ & $\ell$ & $T$ & \# unst. & $\mu^{(\max )} \pm i \omega^{(\max )}$ \\
\hline \multicolumn{9}{|c|}{ symmetry $\left(S, Z_{2}\right)$} \\
\hline \multirow[t]{2}{*}{$\mathrm{LB}$} & & 0.94330 & 1.2137 & 1.551 & & & $1 \mathrm{r}$ & 0.07906 \\
\hline & $S$ & & & & & & +0 & 0.07906 \\
\hline \multirow[t]{2}{*}{ ML } & & 0.88662 & 1.6974 & 1.421 & & & $1 \mathrm{c}$ & $0.02490 \pm i 0.07323$ \\
\hline & $S$ & & & & & & $+1 \mathrm{r}+2 \mathrm{c}$ & $0.2704 \pm i 1.515$ \\
\hline \multirow[t]{2}{*}{ MU } & & 0.87723 & 1.8322 & 1.394 & & & $1 \mathrm{r}$ & 0.05617 \\
\hline & $S$ & & & & & & $+1 \mathrm{r}+2 \mathrm{c}$ & $0.3267 \pm i 1.543$ \\
\hline \multirow[t]{2}{*}{ UB } & & 0.85273 & 2.4990 & 1.298 & & & $3 \mathrm{c}$ & $0.2179 \pm i 1.983$ \\
\hline & $S$ & & & & & & $+6 \mathrm{c}$ & $0.4231 \pm i 1.660$ \\
\hline \multirow[t]{2}{*}{$\mathrm{RPO}_{4.934}$} & & 0.85137 & 2.4451 & 1.302 & 6.423 & 4.934 & $1 c$ & $0.1242 \pm i 0.3819$ \\
\hline & $S$ & & & & & & $+6 \mathrm{c}$ & $0.4417 \pm i 0.3284$ \\
\hline symmetry & $S$ & & & & & & & \\
\hline $\mathrm{S} 2 \mathrm{U}$ & & 0.89383 & 1.4495 & 1.296 & & & $1 \mathrm{c}$ & $0.05592 \pm i 0.5215$ \\
\hline $\mathrm{S} 2 \mathrm{~L}$ & & 0.96159 & 1.1191 & 1.522 & & & $1 \mathrm{r}$ & 0.1090 \\
\hline $\mathrm{RPO}_{10.96}$ & & 0.88845 & 1.5205 & 1.265 & 13.868 & 10.96 & $1 \mathrm{r}+2 \mathrm{c}$ & $0.06051 \pm i 0.15383$ \\
\hline $\mathrm{RPO}_{36.72}$ & & 0.89515 & 1.4865 & 1.291 & 47.417 & 36.72 & $2 \mathrm{r}+5 \mathrm{c}$ & $0.08636 \pm i 0.0900$ \\
\hline ergodic & & 0.8787 & 1.671 & 1.274 & & & & $\approx 0.11$ \\
\hline
\end{tabular}

TABLE 1. All travelling waves and relative periodic orbits studied in this paper for pipe (2.4), split by solution symmetry: (mean) kinetic energy $\bar{E}$; (mean) dissipation $\bar{D}$, both in laminar solution units; travelling wave downstream phase velocity $c$ or relative periodic orbit mean phase velocity $\bar{c}_{p}=\ell_{p} / T_{p}$; accumulated relative periodic orbit shift $\ell_{p}$ (not modulo the periodic cell length $L=5.0265 \ldots$ ); period $T_{p}$; the number of unstable eigen-directions within the solution's symmetry subspace, $\mathrm{r}=$ real, $\mathrm{c}=$ complex; the leading Floquet exponent $\lambda^{(j)}=\mu^{(j)} \pm i \omega^{(j)}$. For the upper part of the table, numbers in $S$ rows are for symmetry-breaking eigenvalues when $Z_{2}$ is removed. 'Ergodic' refers to long time average computed from evolution of typical turbulent states.

plotted in figure $9(b)$. (In absence of a systematic symbolic dynamics, we label $\mathrm{RPO}_{T}$ by its period $T$.)

Several two-dimensional projections of relative periodic orbits $\mathrm{RPO}_{36.72}, \mathrm{RPO}_{10.96}$ and the tiny $\mathrm{RPO}_{4.934}$ are given in figure 11, along with the N2 and S2 states used in this paper (same colour coding as previous plots). Figure 12 (see movie online) shows flow snapshots of $\mathrm{RPO}_{36.72}$ at a fixed axial cross-section. The movie has been taken after reducing the continuous symmetry with the method of the slices and hence shows how the orbit closes after one period. The orbit consists of a slow nearly quiescent phase, during which the neighbourhood of S2U is visited, followed by a period of intense turbulent bursting. This behaviour suggests that the orbit $\mathrm{RPO}_{36.72}$ may be related to a global homoclinic bifurcation off S2U.

\subsection{Discussion}

Symmetry reduction by the method of slices in a high-dimensional flow thus reveals dynamics around recently discovered travelling waves, both local and global, and leads to the discovery of first relative periodic orbits in pipe flow that, as they have been extracted from turbulent trajectories, can be expected to be dynamically important. While the tiny relative periodic orbit $\mathrm{RPO}_{4.934}$ appears to originate from a Hopf bifurcation off a travelling wave very nearby (Duguet et al. 2008; Mellibovsky \& Eckhardt 2011b, have also found similar local relative periodic orbits) $\mathrm{RPO}_{10.96}$ and $\mathrm{RPO}_{36.72}$ exhibit temporal variation typical of the turbulence found in our computational domain (2.4). 


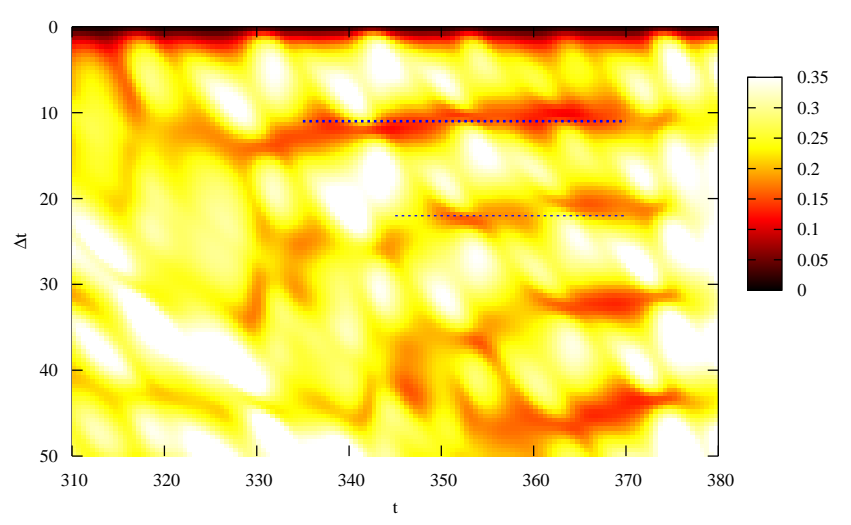

FiguRE 10. Search for recurrences within the slice. Each state is compared with the state at earlier times $\Delta t$ before, shading indicates the relative distance $\|\hat{a}(t)-\hat{a}(t-\Delta t)\|_{c} /\|\hat{a}(t-\Delta t)\|_{c}$. The minima indicated by the horizonal lines suggest that an orbit of period $T \approx 11$ is shadowed for $t \leq 40$ (all times are expressed in units of $D / U$ ). Newton search indeed confirms this, by finding there the weakly unstable $\mathrm{RPO}_{10.96}$. Note that the tiny relative periodic orbit $\mathrm{RPO}_{4.934}$ from figure 11 (a) lies far from the turbulent region, and thus does not show up in recurrence plots.

(a)

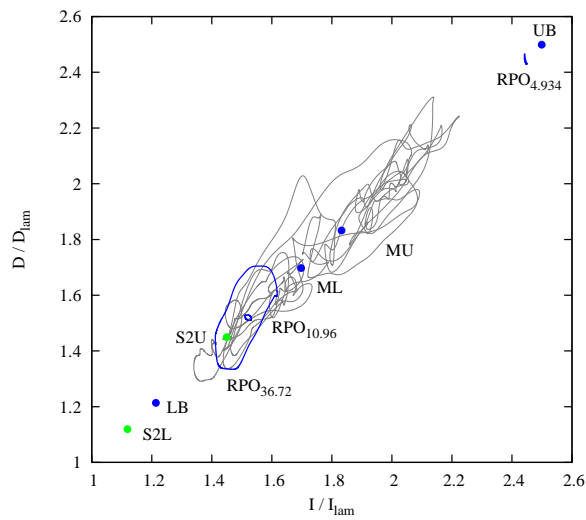

(b)

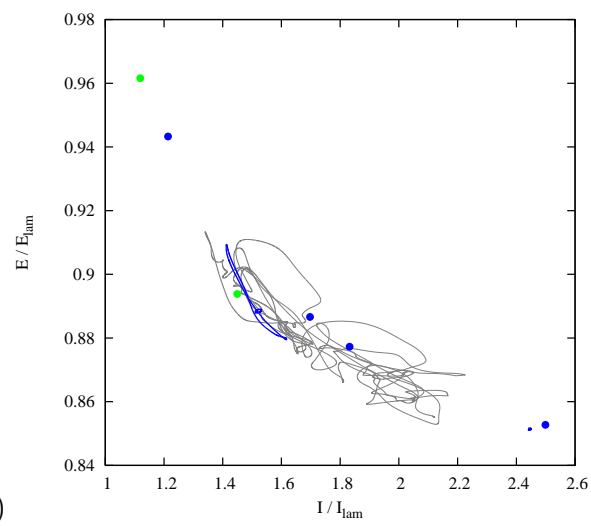

FiguRE 11. Rate of the energy input at the walls $I$ versus (a) the dissipation rate $D$, and (b) the energy $E$, see (2.7). Plotted are all invariant solutions of table 1, together with a typical turbulent orbit. The tiny $\mathrm{RPO}_{4.934}$ is visible as a little twiddle just below UB travelling wave. On the scale of these plots, the Duguet et al. (2008) relative periodic orbit would be indistinguishable from its mother travelling wave.

Visualisations of physical quantities, such as in figure 11, are often used in the literature to infer the importance of coherent solutions (e.g. travelling waves and relative periodic orbits in pipe flow) in turbulent flow. Here the dissipation $D$ and input $I$ of figure 11(a), for example, clearly show that states S2L, LB and UB are far from the turbulent flow. However, the converse is usually not true. As the energy balance (2.7) forces all travelling waves and the averages over all relative periodic orbits, and turbulent flow, to lie on the diagonal $I=D$, travelling waves that feature frictions close to the turbulent average may appear to be in core of the turbulent region. For example, figure 11 suggests that 
20

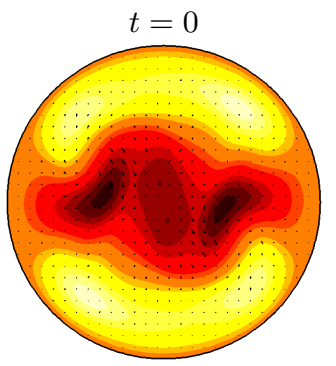

A. P. Willis, P. Cvitanović and M. Avila

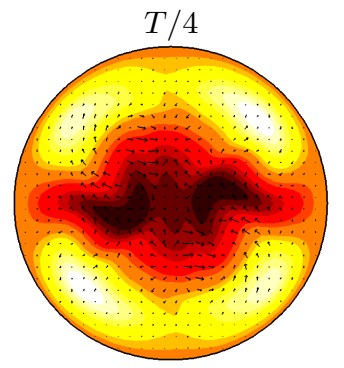

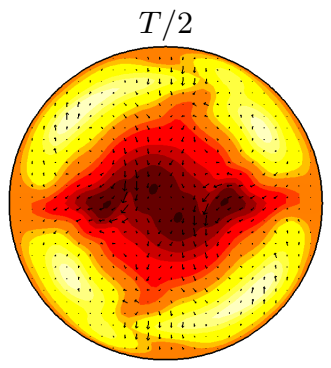

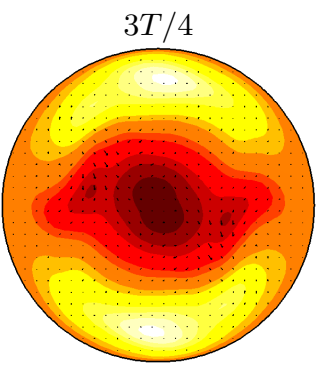

FigURE 12. Four snapshots of relative periodic orbit $\mathrm{RPO}_{36.72}$ at the same fixed pipe section as in (7), reduced by the method of slices into a periodic orbit solution (see movie online). A colormap of stream-wise velocity is shown, with white (black) indicating positive $w=0.6 U$ (negative $w=-0.7 U$ ) velocity with respect to laminar flow. The $t=0$ state has been chosen to be the closest passage (in the energy norm) to travelling wave $\mathrm{S} 2 \mathrm{U}$, see (7).

the ML and MU states may be representative of the turbulent dynamics. The projection within the slice (see figure 9) reveals that in fact these two states, despite having the 'right friction', are far from the turbulent dynamics in phase space. Our results show that the neighbourhoods of known travelling waves (table 1), with the exception of S2U, are visited only for a small fraction of time, in agreement with earlier estimates (Kerswell \& Tutty 2007; Schneider et al. 2007; Willis \& Kerswell 2008). It is only the reduced state space projections that make it quite clear that only $\mathrm{RPO}_{10.96}, \mathrm{RPO}_{36.72}$ and $\mathrm{S} 2 \mathrm{U}$ are embedded in the region associated with turbulence. To sum up, phase-portraits using physically motivated quantities such as dissipation, input and kinetic energy, may be used to rule out the relevance of coherent states in turbulent flow but not to confirm their relevance.

Another important point is that determination of travelling waves by bifurcations and continuation is often physically misleading. Almost all of the previously found travelling waves and nearby tiny relative periodic orbits are highly unstable to perturbations out of their symmetry subspace, highly repelling and not participants in the asymptotic dynamics (see table 1). The exception to this are the nearly laminar lower-branch states, which play a key role organising the dynamics of the turbulent-laminar boundary or edge. Instead, recurrences in turbulent flow used as initial guesses for Newton-Krylov methods allow it to find solutions that are relevant to the turbulent dynamics. Note that the new relative periodic orbits revealed here are associated to the lower dissipation region of turbulent flow; but is expected that application of the method slices together with a systematic study of recurrences in the upper region will yield new 'turbulent' relative periodic orbits and travelling waves.

Finally, it is worth emphasising that restriction of dynamics to flow-invariant subspaces can potentially be very misleading. For example, figure 8 and 9 exhibit completely different dynamics. In this case, imposing the rotate-and-reflect symmetry, in addition to shift-and-reflect, results even in the absence of turbulent dynamics. Hence, despite the similarity of the dynamics of full-space and shift-and-reflect turbulence, our choice may also be problematic.

\section{Conclusion and perspectives}

As a turbulent flow evolves, every so often we catch a glimpse of a familiar structure. For any finite spatial resolution, the flow stays for a finite time in the neighbourhood of a coherent structure belonging to an alphabet of admissible fluid states, represented here by 
a set of travelling wave and relative periodic orbit solutions of Navier-Stokes. These are not the 'modes' of the fluid; they do not provide a decomposition of the flow into a sum of components at different wavelengths, or a basis for low-dimensional modelling. Each such solution spans the whole range of physical scales of the turbulent fluid, from the outer wall-to-wall scale, down to the viscous dissipation scale. Numerical computations require sufficient resolution to cover all of these scales, so no global dimension reduction is likely. The role of invariant solutions of Navier-Stokes is, instead, to partition the $\infty$ dimensional state space into a finite set of neighbourhoods visited by a typical long-time turbulent fluid state.

Motivated by the recent observations of recurrent coherent structures in experimental and numerical turbulent flows, we initiated here an exploration of the hierarchy of travelling waves and relative periodic orbits of fully-resolved transitionally turbulent pipe flow in order to describe its spatio-temporally chaotic dynamics. For pipe flow travelling waves and relative periodic orbits embody a vision of turbulence as a repertoire of recurrent spatio-temporal coherent structures explored by turbulent dynamics. The new relative periodic orbits that we present here are a part of the backbone of this repertoire. Given a set of invariant solutions, the next step is to understand how the dynamics interconnects the neighbourhoods of the invariant solutions discovered so far. Currently, a taxonomy of these myriad states eludes us, but emboldened by successes in applying periodic orbit theory to the simpler Kuramoto-Sivashinsky problem (Christiansen et al. 1997; Lan \& Cvitanović 2008; Cvitanović et al. 2009), we are optimistic.

The reader might rightfully wonder what the short pipe periodic cells studied here and in plane Couette flow have to do with physical, wall-bounded shear flows in general, with large aspect ratios and physical boundary conditions? The $3 D$ fluid states captured by the short pipe invariant solutions and their unstable manifolds are strikingly similar to states observed both in experiments and in numerical simulations of longer pipes (Hof et al. 2004), while the turbulent dynamics visualised in state space appears to be pieced together from close visitations to coherent structures connected by transient interludes. Nevertheless, one of the outstanding issues that must be addressed in future work is the small-aspect cell periodicities imposed for computational efficiency. In case of the pipe flow, most computations of invariant solutions have focused on stream-wise periodic cells barely long enough to allow for sustained turbulence. Such small cells introduce dynamical artifacts such as lack of structural stability and stream-wise cell-size dependence of the sustained turbulence states. Here we can draw inspiration from pattern-formation theory, where the most unstable wavelengths from a continuum of unstable solutions set the scales observed in simulations, with recent progress reported both from the 'microscopic scales' (Schneider et al. 2010), as well as long pipe experiments and phenomenology (Avila et al. 2011).

The main message of this paper is that if a problem has a continuous symmetry, the symmetry must be used to simplify it. Ignore it at your own peril, as has been done earlier in Kuramoto-Sivashinsky (Christiansen et al. 1997) and plane Couette flow (Gibson et al. 2008); the invariant solutions found by restricting searches to the discretesymmetry invariant subspaces have little if anything to do with the full state space explored by turbulence, no more than the equilibrium points of the Lorenz flow have to do with its strange attractor. Note also that the shift of a pipe flow into a slice is not a stream-wise average over the 3D pipe flow. It is the full flow snapshot, embedded in the $\infty$-dimensional state space. Symmetry reduction is not a dimensional-reduction scheme, or flow modelling by fewer degrees of freedom: the reduced state space is also $\infty$-dimensional and no information is lost, one can go freely between solutions in the full and reduced state spaces by integrating the associated reconstruction equations. 
Symmetry reduction by method of slices is numerically efficient. Coupled with our state space visualisations, it allows for explorations of high-dimensional flows that were hitherto unthinkable. Symmetry reduction is here achieved, and now all pipe flow solutions can be plotted together, as one happy family: all points equivalent by symmetries are represented by a single point, families of solutions are mapped to a single solution, travelling waves become equilibria, and relative periodic orbits become periodic orbits. Without symmetry reduction, no full understanding of pipe and plane plane Couette flows is possible.

We would like to acknowledge R. R. Kerswell for providing travelling waves solutions data. We are indebted to R. L. Davidchack, S. Froehlich, B. Hof, and E. Siminos for inspiring discussions, and D. W. Spieker for contributing to the symmetry classification of $\S 2.3$. A. P. W. was initially funded by the European Community's Seventh Framework Programme FP7 2007-2013 under Grant agreement No. PIEF-GA-2008-219-233. M. A. was supported by the Max-Planck-Gesellschaft. P. C. thanks G. Robinson, Jr. for support, and Max-Planck-Institut für Dynamik und Selbstorganisation, Göttingen for hospitality. P. C. was partly supported by NSF grant DMS-0807574 and 2009 Forschungspreis der Alexander von Humboldt-Stiftung.

\section{Appendix A. Discrete symmetries}

In addition to azimuthal reflection, invariant solutions can exhibit further discrete symmetries that derive from azimuthal and stream-wise periodicities over the computational cell (2.3).

Periodicity in the azimuthal direction allows for solutions with discrete cyclic symmetry $g(2 \pi / m, 0)$, defined for integer $m$. Velocity fields invariant under such rational azimuthal shifts are said to be invariant under the discrete cyclic group $\mathrm{C}_{m, \theta}$. Note that all solutions are invariant under $\mathrm{C}_{1, \theta}$, and given the assumed stream-wise periodicity, under $\mathrm{C}_{1, z}$ as well. This permits the study of states in the reduced computational cells $\Omega=[0,1 / 2] \times$ $[0,2 \pi / m] \times[0, \pi / \alpha]$, where $L=\pi / \alpha$. Calculations in larger domains are required to determine subharmonic bifurcations.

Consider states invariant under $\mathrm{C}_{m, \theta}$ and $\mathrm{C}_{1, z}$, and denote half-shifts within our reduced cell, in $\theta$ and $z$ respectively, by $g_{\theta}=g(\pi / m, 0)$ and $g_{z}=g(0, L / 2)$. For the special case of a half-shift in azimuth, $\sigma$ and $g_{\theta}$ commute so that

$$
G=\mathrm{D}_{1} \times \mathrm{C}_{m, \theta} \times \mathrm{C}_{1, z} \subset \Gamma
$$

is abelian and of order 8 ,

$$
G=\left\{e, g_{\theta}, g_{z}, g_{\theta} g_{z}, \sigma, \sigma g_{\theta}, \sigma g_{z}, \sigma g_{\theta} g_{z}\right\} .
$$

Focus lies on the following subgroups:

$$
Z=\{e, \sigma\}, \quad S=\left\{e, \sigma g_{z}\right\}, \quad \Omega_{m}=\left\{e, g_{\theta} g_{z}\right\} .
$$

The first is the 'reflectional', or 'mirror' symmetry, the second is the 'shift-and-reflect' symmetry, and the third is the 'shift-and-rotate' symmetry. States invariant under $g_{\theta}$ or $g_{z}$ are invariant under $\mathrm{C}_{2 m, \theta}$ or $\mathrm{C}_{2, z}$ and hence become redundant upon redefinition $m:=2 m$ or $\alpha:=2 \alpha$ (i.e. they reduce to half-cells). It can also be shown that $\sigma g_{\theta}=g_{\theta}^{-1 / 2} \sigma g_{\theta}^{1 / 2}$, where $g_{\theta}^{1 / 2}$ is the half-half-shift, and therefore that $\sigma g_{\theta} g_{z}=g_{\theta}^{-1 / 2} \sigma g_{z} g_{\theta}^{1 / 2}$. Invariance under these combinations is conjugate to $Z$ and $S$. We use, however, the 'rotate-andreflect' subgroup, denoted by

$$
Z_{m}=\left\{e, \sigma g_{\theta}\right\}
$$


which has mirror reflection planes located at $\theta= \pm \pi /(2 m)$ (see figure 7 for the case $m=2$ ).

The first travelling waves found for pipe flow were invariant under $S$ and $\mathrm{C}_{m, \theta}$ for $m=2,3,4, \ldots$ (Faisst \& Eckhardt 2003; Wedin \& Kerswell 2004). More recently the 'missing' $m=1$ state has been located (Pringle \& Kerswell 2007) and many more states invariant under more than one of the above classes (Pringle et al. 2009). States invariant under $(S, Z)$ implies invariance under $\sigma \sigma g_{z}=g_{z}$, and hence under $\mathrm{C}_{2, z}$, reducing to the half-length pipe. Invariance under $\left(S, \Omega_{m}\right)$ is permissible, however, and using the combinations above it can be calculated that $\left(S, \Omega_{m}\right)=\left(S, Z_{m}\right)=\left(Z_{m}, \Omega_{m}\right)$. Such states have been termed 'highly symmetric' by Pringle et al. (2009). As reflection is arguably easier to visualise than shift-and-rotate, we use the notation $\left(S, Z_{m}\right)$ for these states.

\section{REFERENCES}

Auerbach, D., Cvitanović, P., Eckmann, J.-P., Gunaratne, G. \& Procaccia, I. 1987 Exploring chaotic motion through periodic orbits. Phys. Rev. Lett. 58, 23.

Avila, K., Moxey, D., De lozar, A., Avila, M., Barkley, D. \& Hof, B. 2011 The onset of turbulence in pipe flow. Science 333, 192-196.

Avila, M., Willis, A. \& Hof, B. 2010 On the transient nature of localized pipe flow turbulence. J. Fluid Mech. 646, 127-136.

Beyn, W.-J. \& ThümmleR, V. 2004 Freezing solutions of equivariant evolution equations. SIAM J. Appl. Dyn. Syst. 3, 85-116.

CARTAN, E. 1935 La méthode du repère mobile, la théorie des groupes continus, et les espaces généralisés, Exposés de Géométrie, vol. 5. Paris: Hermann.

Christiansen, F., Cvitanović, P. \& Putkaradze, V. 1997 Spatio-temporal chaos in terms of unstable recurrent patterns. Nonlinearity 10, 55-70, arXiv: chao-dyn/9606016.

Cvitanović, P. 2007 Continuous symmetry reduced trace formulas. ChaosBook. org/ predrag/papers/trace.pdf.

Cvitanović, P., Artuso, R., Mainieri, R., Tanner, G. \& Vattay, G. 2011 Chaos: Classical and Quantum. Copenhagen: Niels Bohr Inst., ChaosBook.org.

Cvitanović, P., Davidchack, R. L. \& Siminos, E. 2009 On the state space geometry of the Kuramoto-Sivashinsky flow in a periodic domain. SIAM J. Appl. Dyn. Syst. 9, 1-33, arXiv: 0709.2944.

Cvitanović, P. \& Gibson, J. F. 2010 Geometry of turbulence in wall-bounded shear flows: Periodic orbits. Phys. Scr. T 142, 014007.

Duguet, Y., Pringle, C. C. T. \& Kerswell, R. R. 2008 Relative periodic orbits in transitional pipe flow. Phys. Fluids 20, 114102, arXiv:0807.2580.

Faisst, H. \& Eckhardt, B. 2003 Traveling waves in pipe flow. Phys. Rev. Lett. 91, 224502.

Fels, M. \& Olver, P. J. 1998 Moving coframes: I. A practical algorithm. Acta Appl. Math. $\mathbf{5 1}, 161-213$.

Fels, M. \& Olver, P. J. 1999 Moving coframes: II. Regularization and theoretical foundations. Acta Appl. Math. 55, 127-208.

Frisch, U. 1996 Turbulence. Cambridge, UK: Cambridge Univ. Press.

Froenlich, S. \& Cvitanović, P. 2011 Reduction of continuous symmetries of chaotic flows by the method of slices. Comm. Nonlinear Sci. and Numerical Simulation 17, 2074-2084, arXiv:1101.3037.

Gibson, J. F., Halcrow, J. \& Cvitanović, P. 2008 Visualizing the geometry of state space in plane Couette flow. J. Fluid Mech. 611, 107-130, arXiv:0705.3957.

Gibson, J. F., Halcrow, J. \& Cvitanović, P. 2009 Equilibrium and traveling-wave solutions of plane Couette flow. J. Fluid Mech. 638, 1-24, arXiv:0808.3375.

Greene, J. M. \& Kim, J.-S. 1988 The steady states of the Kuramoto-Sivashinsky equation. Physica D 33, 99-120.

Halcrow, J., Gibson, J. F., Cvitanović, P. \& Viswanath, D. 2009 Heteroclinic connections in plane Couette flow. J. Fluid Mech. 621, 365-376, arXiv:0808.1865. 
Hamilton, J. M., Kim, J. \& Waleffe, F. 1995 Regeneration mechanisms of near-wall turbulence structures. J. Fluid Mech. 287, 317-348.

Hof, B., De Lozar, A., Kuik, D. \& Westerweel, J. 2008 Repeller or attractor? Selecting the dynamical model for the onset of turbulence in pipe flow. Phys. Rev. Lett. 101, 214501.

Hof, B., van Doorne, C. W. H., Westerweel, J., Nieuwstadt, F. T. M., Faisst, H., Eckhardt, B., Wedin, H., Kerswell, R. R. \& Waleffe, F. 2004 Experimental observation of nonlinear traveling waves in turbulent pipe flow. Science 305, 1594-1598.

Hopf, E. 1948 A mathematical example displaying features of turbulence. Comm. Pure Appl. Math. 1, 303-322.

Huygens, C. 1673 L'Horloge à Pendule. Amsterdam: Swets \& Zeitlinger.

Kawahara, G. \& KidA, S. 2005 Elementary coherent structures in turbulent flows. Parity 20, 12.

Kerswell, R. R. \& Tutty, O. 2007 Recurrence of travelling waves in transitional pipe flow. J. Fluid Mech. 584, 69-102, arXiv:physics/0611009.

Kim, K. C. \& Adrian, R. J. 1999 Very large-scale motion in the outer layer. Phys. Fluids 11, 417-422.

Kline, S. J., Reynolds, W. C., Schraub, F. A. \& Runstadler, P. W. 1967 The structure of turbulent boundary layers. J. Fluid Mech. 30, 741-773.

Lan, Y. \& Cvitanović, P. 2008 Unstable recurrent patterns in Kuramoto-Sivashinsky dynamics. Phys. Rev. E 78, 026208, arXiv.org:0804.2474.

Lombardi, M., Caulfield, C., Cossu, C., Pesci, A. \& Goldstein, R. 2011 Growth and instability of a laminar plume in a strongly stratified environment. J. Fluid Mech.671, $184-206$.

Mellibovsky, F. \& Eckhardt, B. 2011a From travelling waves to mild chaos: a supercritical bifurcation cascade in pipe flow. Arxiv preprint arXiv:1110.1222 .

Mellibovsky, F. \& Eckhardt, B. $2011 b$ Takens-Bogdanov bifurcation of travelling-wave solutions in pipe flow. J. Fluid Mech. 670, 96-129.

Mullin, T. \& Kerswell, R. R. 2005 Non-uniqueness of Solutions to the Navier-Stokes Equations and their Connection with Laminar-Turbulent Transition. Dordrecht: Kluwer.

Olver, P. J. 1999 Classical Invariant Theory. Cambridge: Cambridge Univ. Press.

Poincaré, H. 1896 Sur les solutions périodiques et le principe de moindre action. C. R. Acad. Sci. Paris 123, 915-918.

Pringle, C. C. T., Duguet, Y. \& Kerswell, R. R. 2009 Highly symmetric travelling waves in pipe flow. Phil. Trans. Royal Soc. A 367, 457-472, arXiv:0804.4854.

Pringle, C. C. T. \& Kerswell, R. R. 2007 Asymmetric, helical, and mirror-symmetric traveling waves in pipe flow. Phys. Rev. Lett. 99, 074502.

RAND, D. 1982 Dynamics and symmetry - predictions for modulated waves in rotating fluids. Arch. Rational Mech. Anal. 79, 1-3.

Recke, L., Samollenko, A., Tkachenko, V. \& Yanchuk, S. 2011 Frequency locking by external forcing in systems with rotational symmetry. arXiv:1108.5990.

Rowley, C. W. \& Marsden, J. E. 2000 Reconstruction equations and the Karhunen-Loéve expansion for systems with symmetry. Physica D 142, 1-19.

Schneider, T. M., Eckhardt, B. \& Vollmer, J. 2007 Statistical analysis of coherent structures in transitional pipe flow. Phys. Rev. E 75, 066313.

Schneider, T. M., Gibson, J. F. \& Burke, J. 2010 Snakes and ladders: Localized solutions of plane Couette flow. Phys. Rev. Lett. 104, 104501.

Siminos, E. \& Cvitanović, P. 2011 Continuous symmetry reduction and return maps for high-dimensional flows. Physica D 240, 187-198.

Tempelmann, D., Hanifi, A. \& Henningson, D. S. 2010 Spatial optimal growth in threedimensional boundary layers. J. Fluid Mech. 646, 5-37.

Viswanath, D. 2007 Recurrent motions within plane Couette turbulence. J. Fluid Mech. 580, 339-358, arXiv:physics/0604062.

Wedin, H. \& Kerswell, R. R. 2004 Exact coherent structures in pipe flow: Traveling wave solutions. J. Fluid Mech. 508, 333-371.

Willis, A. \& Kerswell, R. 2008 Coherent structures in localised and global pipe turbulence. Phys. Rev. Lett. 100, 124501. 